\title{
$N$-step energy of maps and the fixed-point property of random groups
}

\author{
Hiroyasu Izeki, Takefumi Kondo and Shin Nayatani
}

\begin{abstract}
We prove that a random group of the graph model associated with a sequence of expanders has the fixed-point property for a certain class of CAT(0) spaces. We use Gromov's criterion for the fixed-point property in terms of the growth of $n$-step energy of equivariant maps from a finitely generated group into a CAT( 0$)$ space, for which we give a detailed proof. We estimate a relevant geometric invariant of the tangent cones of the Euclidean buildings associated with the groups PGL $\left(m, \mathbb{Q}_{r}\right)$, and deduce from the general result above that the same random group has the fixed-point property for all of these Euclidean buildings with $m$ bounded from above.
\end{abstract}

Mathematics Subject Classification (2010). 20F65, 58E20, $20 \mathrm{P05.}$

Keywords. Finitely generated group, random group, CAT(0) space, fixed-point property, energy of map, Wang invariant, expander, Euclidean building.

\section{Introduction}

Random groups were introduced by Gromov [7] as a framework in which he justified his previous claim that 'most' discrete groups are hyperbolic [6]. While this standard model, called the density model, of random groups has been actively studied, Gromov [8] introduced another model, called the graph model, of random groups in search for infinite groups that cannot be uniformly embedded into Hilbert spaces, thereby being a counterexample to a version of Baum-Connes conjecture. Note that the graph model is formed by choosing an infinite sequence of finite graphs with increasing vertices, and Gromov chose a sequence of (bounded-degree) expanders satisfying some additional conditions so that the corresponding random group was non-elementary hyperbolic, hence infinite. Throughout the introduction, we assume that this choice is made and fixed. In the same paper, Gromov claimed that a random group of the graph model had the fixed-point property for all Hadamard manifolds (possibly of infinite dimensions). Here we say that a group $\Gamma$ has the fixed-point property for a metric space $Y$ if for any homomorphism $\rho: \Gamma \rightarrow \operatorname{Isom}(Y), \rho(\Gamma)$ has a global fixed point in $Y$. If $\mathcal{Y}$ is a family of metric spaces and $\Gamma$ has the fixed-point property for all 
members of $\mathcal{Y}$, we say that $\Gamma$ has the fixed-point property for $\mathcal{Y}$. Silberman [21] then rigorously proved that the same random group had the fixed-point property for Hilbert spaces, which is equivalent to saying that the random group had Kazhdan's property (T). We refer the reader to Ollivier's monograph [20] for extensive information on random groups.

In the present paper, we prove that a random group of the graph model has the fixedpoint property for a certain class of CAT( 0 ) spaces, including all Hadamard manifolds. We therefore justify the above mentioned claim of Gromov in a generalized form. To state our main result more in detail, recall that we [11] introduced a certain geometric invariant, denoted by $\delta$, of $\operatorname{CAT}(0)$ space which takes values in the interval $[0,1]$. It is worth mentioning that the invariant of a $\operatorname{CAT}(0)$ space can be computed as the supremum of its values for all tangent cones of the space. For $0 \leq \delta_{0}<1$, let $y_{\leq \delta_{0}}$ denote the class of CAT(0) spaces $Y$ satisfying $\delta(Y) \leq \delta_{0}$, or equivalently $\delta\left(T C_{p} Y\right) \leq \delta_{0}$ for all $p \in Y$. Then a random group is infinite hyperbolic and has the fixed-point property for all members of $y_{\leq \delta_{0}}$. This result is compared to the authors' previous result that if $\delta_{0}<1 / 2$, a random group in Zuk's triangular model has the fixed-point property for all members of $y_{\leq \delta_{0}}$.

As in Silberman's, our proof is built of two parts, one geometric and the other probabilistic. The probabilistic part follows Silberman's argument mostly verbatim, but our presentation has some advantages. First, we simplify his argument by replacing the large deviation inequality for the Bernoulli walk he used by the central limit theorem. This also enables us to allow degree two vertices in the graphs and therefore state our result in a form applicable to subdivided expanders. Secondly, we generalize Silberman's spectral gap inequality for maps from a finite graph into a Hilbert space to the inequality for maps with $\mathrm{CAT}(0)$ targets, and this enables us to state the result for more general CAT(0) spaces than Hilbert spaces. The geometric part of the proof is completely different from Silberman's; we use Gromov's criterion for the fixed-point property in terms of the growth of $n$-step energy of equivariant maps from a group into a CAT(0) space. Since Gromov does not give a detailed proof to this result, we undertake to do so.

With the general result at hand, it is important to compute or estimate from above the invariant $\delta$ of a $\operatorname{CAT}(0)$ metric cone. To do this, we relate it to a modified version of distortion of the cone, which we call the radial distortion.

The following fact is well known. Suppose that a discrete group $\Gamma$ has the fixedpoint property for Hilbert spaces, all symmetric spaces associated with the groups $\operatorname{PGL}(m, \mathbb{R})$ and $\operatorname{PGL}(m, \mathbb{C})$, and all Euclidean buildings associated with the groups $\operatorname{PGL}\left(m, \mathbb{Q}_{r}\right)$ with $r$ prime. Then $\Gamma$ is nonlinear, in the sense that it admits no faithful linear representation, and more strongly, any finite-dimensional linear representation of $\Gamma$ has finite image. It is therefore interesting to see which of these spaces are in the above class of CAT( 0 ) spaces. Since Hilbert spaces and the symmetric spaces are Hadamard manifolds and hence trivially belong to this class, it remains to investigate the Euclidean buildings. As mentioned above, the estimation of the invariant $\delta$ of their tangent cones can be reduced to that of the radial distortion of these cones. By 
carrying out the latter task, we conclude that the invariant $\delta$ of the tangent cones of the Euclidean buildings are bounded from above by a constant less than one which depends only on the dimensions of the buildings. Combining this and the general result above, we finally conclude that a random group of the graph model has the fixed-point property for all of the Euclidean buildings with dimensions bounded from above by a positive integer, specified in advance.

This paper is organized as follows. In Section 1 we prepare some definitions, notations and results concerning CAT(0) spaces which are necessary in later sections. In Section 2 under the situation that an isometric action of a finitely generated group on a CAT(0) space is given, we study the $n$-step energy of an equivariant map from the group into the CAT(0) space. After some preliminaries, we give a proof to Gromov's result which states that if the $n$-step energy grows strictly slower than $n$ times the (single-step) energy, then the action is forced to have a global fixed point. In fact, we prove the result in a slightly generalized form, which will be necessary in the next section. We then treat the special case that the target space is an affine Hilbert space, and conclude this section by proving a sort of converse of Gromov's result holds for some CAT(0) spaces. In Section 3 we first recall the formalism of the graph model of random groups, and then prove a fixed-point theorem for random groups, a version of which will be stated in terms of the invariant $\delta$. In Section 4 we first give an upper bound for the invariant $\delta$ of a $\mathrm{CAT}(0)$ metric cone, restricted to measures with barycenter at the cone point, in terms of the radial distortion of the cone. We then estimate $\delta$ of the tangent cones of the Euclidean buildings associated with the groups PGL $\left(m, \mathbb{Q}_{r}\right)$ by determining the radial distortion of the cones.

Part of this paper was announced in [9], [13].

\section{Preliminaries on CAT(0) spaces}

In this section, we briefly recall some definitions and results concerning CAT(0) spaces. We refer the reader to [2] for a detailed exposition on the subject. We follow the notations used in [11], $\S 1$.

Let $Y$ be a metric space and $p, q \in Y$. A geodesic joining $p$ to $q$ is a map $c:[0, l] \rightarrow Y$ satisfying $c(0)=p, c(l)=q$ and $d\left(c(t), c\left(t^{\prime}\right)\right)=\left|t-t^{\prime}\right| d(p, q) / l$ for any $t, t^{\prime} \in[0, l]$. A geodesic $c:[0, l] \rightarrow Y$ with $l=d(c(0), c(l))$ is called unit speed; a unit speed geodesic is nothing but an isometric embedding of an interval. We say that $Y$ is a geodesic space if any two points in $Y$ are joined by a geodesic.

Consider a triangle in $Y$ whose vertices are $p_{1}, p_{2}, p_{3} \in Y$ and sides are three geodesic segments $p_{1} p_{2}, p_{2} p_{3}, p_{3} p_{1}$ joining pairs of these vertices. We denote this triangle by $\Delta\left(p_{1}, p_{2}, p_{3}\right)$ and call such a triangle a geodesic triangle. Take a triangle $\Delta\left(\overline{p_{1}}, \overline{p_{2}}, \overline{p_{3}}\right)$ in $\mathbb{R}^{2}$ with the same side lengths: $d_{\mathbb{R}^{2}}\left(\overline{p_{i}}, \overline{p_{j}}\right)=d_{Y}\left(p_{i}, p_{j}\right)$. We call $\Delta\left(\overline{p_{1}}, \overline{p_{2}}, \overline{p_{3}}\right)$ a comparison triangle for $\Delta\left(p_{1}, p_{2}, p_{3}\right)$. A point $\bar{q} \in \overline{p_{i}} \overline{p_{j}}$ is called a comparison point for $q \in p_{i} p_{j}$ if $d_{Y}\left(p_{i}, q\right)=d_{\mathbb{R}^{2}}\left(\overline{p_{i}}, \bar{q}\right)$. A geodesic triangle $\Delta\left(p_{1}, p_{2}, p_{3}\right)$ in $Y$ is said to satisfy the CAT $(0)$ condition if $d_{Y}\left(q_{1}, q_{2}\right) \leq d_{\mathbb{R}^{2}}\left(\overline{q_{1}}, \overline{q_{2}}\right)$ 
for any pair of points $q_{1}, q_{2}$ on the sides of $\Delta\left(p_{1}, p_{2}, p_{3}\right)$ and their comparison points $\overline{q_{1}}, \overline{q_{2}}$. A geodesic space $Y$ is called a CAT( 0$)$ space if every geodesic triangle in $Y$ satisfies the CAT( 0$)$ condition. Roughly speaking, a CAT(0) space is a geodesic space all of whose geodesic triangles are thinner than Euclidean triangles.

Note that, for a CAT(0) space $Y$, the uniqueness of a unit speed geodesic joining any pair of points in $Y$, and the contractibility of $Y$ immediately follow from the definition. Throughout this paper, we assume metric spaces under consideration are complete. (We should point out here that a complete CAT(0) space was given a distinguished name 'Hadamard space' in [11], though we will not use this terminology in the present paper.)

The following is a characterization of $\operatorname{CAT}(0)$ spaces, which is often used as an alternative definition of $\mathrm{CAT}(0)$ spaces.

Proposition 1.1 ([2]). A geodesic space $Y$ is a CAT(0) space if and only if, for any $p \in Y$ and any geodesic $c:[0,1] \rightarrow Y$,

$$
d(p, c(t))^{2} \leq(1-t) d(p, c(0))^{2}+t d(p, c(1))^{2}-t(1-t) d(c(0), c(1))^{2}
$$

holds.

Proposition 1.2. Let $Y$ be a $\mathrm{CAT}(0)$ space, and $v$ a probability measure on $Y$. Suppose that the integral

$$
\int_{Y} d(p, q)^{2} d v(p)
$$

is finite for some (hence any) point $q \in Y$. Then there exists a unique point $p_{0} \in Y$ which minimizes the function

$$
q \mapsto \int_{Y} d(p, q)^{2} d v(p), \quad q \in Y .
$$

For a proof, see [15], p. 639, Lemma 2.5.1. We call the point $p_{0}$ the barycenter of $v$ and denote it by $\bar{v}$ or $\operatorname{bar}(v)$. Mostly, we will consider a measure $v$ with finite support; $v$ is given as a convex combination $v=\sum_{i=1}^{m} t_{i} \operatorname{Dirac}_{p_{i}}$ of Dirac measures $\operatorname{Dirac}_{p_{i}}$ 's, where $\sum_{i=1}^{m} t_{i}=1$ and $t_{i} \geq 0$ for $i=1, \ldots, m$. In such a case, we often say $\bar{v}$ is the barycenter of $\left\{p_{1}, \ldots, p_{m}\right\}$ with weight $\left\{t_{1}, \ldots, t_{m}\right\}$.

Definition 1.3. Let $Y$ be a CAT(0) space.

(1) Let $c$ and $c^{\prime}$ be two nontrivial geodesics in $Y$ starting from $p \in Y$. The angle $\angle_{p}\left(c, c^{\prime}\right)$ between $c$ and $c^{\prime}$ is defined by

$$
\angle_{p}\left(c, c^{\prime}\right)=\lim _{t, t^{\prime} \rightarrow 0} \angle_{\bar{p}}(\overline{c(t)}, \overline{c(t)}),
$$

where $\angle_{\bar{p}}(\overline{c(t)}, \overline{c(t)})$ denotes the angle between the sides $\bar{p} \overline{c(t)}$ and $\bar{p} \overline{c^{\prime}(t)}$ of the comparison triangle $\Delta(\bar{p}, \overline{c(t)}, \overline{c(t)}) \subset \mathbb{R}^{2}$. 
(2) Let $p \in Y$. We define an equivalence relation $\sim$ on the set of nontrivial geodesics starting from $p$ by $c \sim c^{\prime} \Longleftrightarrow L_{p}\left(c, c^{\prime}\right)=0$. Then the angle $L_{p}$ induces a distance on the quotient $\left(S_{p} Y\right)^{\circ}=$ nontrivial geodesics starting from $\left.p\right\} / \sim$, which we denote by the same symbol $L_{p}$. The completion $\left(S_{p} Y, L_{p}\right)$ of the metric space $\left(\left(S_{p} Y\right)^{\circ}, L_{p}\right)$ is called the space of directions at $p$.

(3) Let $T C_{p} Y$ be the cone over $S_{p} Y$, namely,

$$
T C_{p} Y=\left(S_{p} Y \times \mathbb{R}_{+}\right) /\left(S_{p} Y \times\{0\}\right) .
$$

Let $v, v^{\prime} \in T C_{p} Y$. We may write $v=(u, t)$ and $v^{\prime}=\left(u^{\prime}, t^{\prime}\right)$, where $u, u^{\prime} \in S_{p} Y$ and $t, t^{\prime} \in \mathbb{R}_{+}$. Then

$$
d_{T C_{p} Y}\left(v, v^{\prime}\right)=t^{2}+t^{\prime 2}-2 t t^{\prime} \cos \angle_{p}\left(u, u^{\prime}\right)
$$

defines a distance on $T C_{p} Y$. The metric space $\left(T C_{p} Y, d_{T C_{p} Y}\right)$ is again a $\operatorname{CAT}(0)$ space and is called the tangent cone of $Y$ at $p$. We define an 'inner product' on $T C_{p} Y$ by

$$
\left\langle v, v^{\prime}\right\rangle=t t^{\prime} \cos \angle_{p}\left(u, u^{\prime}\right) .
$$

We often denote the length $t$ of $v$ by $|v|$; thus we have $|v|=\sqrt{\langle v, v\rangle}=d_{T C_{p} Y}\left(0_{p}, v\right)$, where $0_{p}$ denotes the cone point, which is the equivalence class of $(u, 0) \in S_{p} Y \times \mathbb{R}_{+}$ in $T C_{p} Y$.

(4) Define a map $\pi_{p}: Y \rightarrow T C_{p} Y$ by $\pi_{p}(q)=\left([c], d_{Y}(p, q)\right)$, where $c$ is the geodesic joining $p$ to $q$ and $[c] \in S_{p} Y$ is the equivalence class of $c$. Then $\pi_{p}$ is distance-nonincreasing.

A complete, simply connected Riemannian manifold $Y$ with nonpositive sectional curvature, often called a Hadamard manifold, is a typical example of CAT(0) space. For such a $Y, S_{p} Y$ (resp. $T C_{p} Y$ ) is the unit tangent sphere (resp. the tangent space) at $p$. The map $\pi_{p}$ is the inverse of the exponential map. Hilbert spaces, metric trees and Euclidean buildings supply other examples of CAT( 0$)$ spaces (see $\$ 4$ for Euclidean buildings).

\section{The $n$-step energy of equivariant maps}

Let $\Gamma$ be a finitely generated group, and $Y$ a CAT(0) space. Suppose that a homomorphism $\rho: \Gamma \rightarrow \operatorname{Isom}(Y)$ is given. In [8], Gromov formulated a sufficient condition for $\rho(\Gamma)$ to have a global fixed point (i.e., there exists a point $p \in Y$ such that $\rho(\Gamma) p=p$ ) in terms of the growth of $n$-step energy of $\rho$-equivariant maps. The purpose of this section is to give a detailed proof of Gromov's result.

We consider a random walk on $\Gamma$ given by transition probability measures $\{\mu(\gamma, \cdot)\}_{\gamma \in \Gamma}$ on $\Gamma$ which is $\Gamma$-invariant, finitely supported, symmetric, and irreducible. In other words, we are given a nonnegative function $\mu$ on $\Gamma \times \Gamma$ satisfying 
(a) $\mu\left(\gamma \gamma^{\prime}, \gamma \gamma^{\prime \prime}\right)=\mu\left(\gamma^{\prime}, \gamma^{\prime \prime}\right)$ for any $\gamma, \gamma^{\prime}$, and $\gamma^{\prime \prime} \in \Gamma$,

(b) for any $\gamma \in \Gamma, \mu\left(\gamma, \gamma^{\prime}\right)=0$ for all but finitely many $\gamma^{\prime} \in \Gamma$,

(c) for any $\gamma \in \Gamma, \sum_{\gamma^{\prime} \in \Gamma} \mu\left(\gamma, \gamma^{\prime}\right)=1$,

(d) $\mu\left(\gamma, \gamma^{\prime}\right)=\mu\left(\gamma^{\prime}, \gamma\right)$ for any $\gamma, \gamma^{\prime} \in \Gamma$,

(e) for any $\gamma, \gamma^{\prime} \in \Gamma$, there exist $\gamma_{0}, \gamma_{1}, \ldots, \gamma_{n} \in \Gamma$ such that $\gamma=\gamma_{0}, \gamma^{\prime}=\gamma_{n}$, and $\mu\left(\gamma_{i}, \gamma_{i+1}\right) \neq 0, i=0, \ldots, n-1$.

The last condition is called the irreducibility of a random walk and means that $\Gamma$ is 'connected' with respect to $\mu$, that is, for any pair of points in $\Gamma$, one can move from one to the other with positive probability. Though we could begin with a discrete countable group $\Gamma$, the existence of such a $\mu$ would force $\Gamma$ to be finitely generated.

We say a map $f: \Gamma \rightarrow Y$ is $\rho$-equivariant if $f$ satisfies $f\left(\gamma \gamma^{\prime}\right)=\rho(\gamma) f\left(\gamma^{\prime}\right)$ for all $\gamma, \gamma^{\prime} \in \Gamma$. (Here we regard $\Gamma$ itself as a space with left $\Gamma$-action.) We define the energy $E_{\mu, \rho}(f)$ of a $\rho$-equivariant map $f$ by

$$
E_{\mu, \rho}(f)=\frac{1}{2} \sum_{\gamma^{\prime} \in \Gamma} \mu\left(\gamma, \gamma^{\prime}\right) d_{Y}\left(f(\gamma), f\left(\gamma^{\prime}\right)\right)^{2},
$$

where $\gamma$ is an arbitrarily chosen element of $\Gamma$. Note that since $f$ is $\rho$-equivariant and $\mu$ is $\Gamma$-invariant, the right-hand side of (2.1) does not depend on the particular choice of $\gamma$. It is often convenient to choose $\gamma=e$, the identity element of $\Gamma$. A $\rho$-equivariant map $f$ is said to be harmonic if $f$ minimizes $E_{\mu, \rho}$ among all $\rho$-equivariant maps. Note that the image of a $\rho$-equivariant map $f: \Gamma \rightarrow Y$ is the $\rho(\Gamma)$-orbit of the point $f(e)$, and $f$ is determined by the choice of $f(e) \in Y$. Therefore, the set of all $\rho$-equivariant maps from $\Gamma$ to $Y$, denoted by $\mathcal{M}_{\rho}$, can be identified with $Y$. Then the energy functional $E_{\mu, \rho}$ becomes a convex continuous function on $\mathcal{M}_{\rho} \cong Y$.

We define the link $L_{\gamma}$ of $\gamma \in \Gamma$ with respect to $\mu$ by $L_{\gamma}=\left\{\gamma^{\prime} \in \Gamma \mid \mu\left(\gamma, \gamma^{\prime}\right)>\right.$ $0\}$, and for a $\rho$-equivariant map $f$, define a map $F_{\gamma}: L_{\gamma} \rightarrow T C_{f(\gamma)} Y$ by $F_{\gamma}\left(\gamma^{\prime}\right)=$ $\pi_{f(\gamma)}\left(f\left(\gamma^{\prime}\right)\right)$, where $T C_{p} Y$ is the tangent cone of $Y$ at $p$ and $\pi_{p}: Y \rightarrow T C_{p} Y$ is the natural projection. Denote by $-\Delta_{\mu} f(e) \in T C_{f(e)} Y$ the barycenter of the pushforward measure $\left(F_{e}\right)_{*}(\mu(e, \cdot))$. Then a $\rho$-equivariant map $f$ is harmonic if and only if $-\Delta_{\mu} f(e)=0_{f(e)}$. Note that $2\left(-\Delta_{\mu} f(e)\right)$ should be interpreted as the negative of the gradient of $E_{\mu, \rho}$ at $f$. (Indeed, they coincide when $Y$ is a Riemannian manifold. See [10], [11].)

The following proposition gives a sufficient condition for the existence of a fixedpoint of $\rho(\Gamma)$ in terms of the energy functional.

Proposition 2.1 ([10], [11]). Let $\Gamma$ be a finitely generated group equipped with a $\Gamma$-invariant, finitely supported, symmetric, and irreducible random walk $\mu$. Let $Y$ be a $\mathrm{CAT}(0)$ space and $\rho: \Gamma \rightarrow \operatorname{Isom}(Y)$ a homomorphism. Suppose that there is a positive constant $C$ such that $\left|-\Delta_{\mu} f(e)\right|^{2} \geq C E_{\mu, \rho}(f)$ holds for every $\rho$ equivariant map $f$. Then $\rho(\Gamma)$ admits a global fixed point. 
In fact, under the assumption, $\left|-\Delta_{\mu} f_{t}(e)\right|$ decreases to 0 rapidly along the JostMayer gradient flow $f_{t}$ of $E_{\mu, \rho}$, and is integrable on [0, $\infty$ ). (See [12] and [18] for the Jost-Mayer gradient flow.) This means that the length of the flow starting from $f_{0}=f$ is finite up to time infinity. In particular, by taking a divergent sequence $\left\{t_{i}\right\}_{i \in \mathbb{N}} \subset \mathbb{R}$, we obtain a Cauchy sequence $\left\{f_{t_{i}}\right\}_{i \in \mathbb{N}} \subset \mathcal{M}_{\rho}$ and a $\rho$-equivariant map $f_{\infty}$ as its limit. Since $\left|-\Delta_{\mu} f_{t}(e)\right| \rightarrow 0$, under the assumption again, we see that $f_{\infty}$ satisfies $E_{\mu, \rho}\left(f_{\infty}\right)=0$, which implies that $d\left(f(\gamma), f\left(\gamma^{\prime}\right)\right)=0$ whenever $\mu\left(\gamma, \gamma^{\prime}\right) \neq 0$. Since $\mu$ is irreducible, any pair of elements in $\Gamma$ can be connected by a path consisting of segments of the form $\left(\gamma, \gamma^{\prime}\right)$ such that $\mu\left(\gamma, \gamma^{\prime}\right) \neq 0$. Therefore, $f_{\infty}$ must be a constant map. (Actually, the irreducibility of $\mu$ is necessary only at this point.) Since $f_{\infty}(\Gamma)$ is a $\rho(\Gamma)$-orbit consisting of a single point, it is fixed by $\rho(\Gamma)$.

For $\mu$ as above, denote by $\mu^{n}$ the $n$th convolution of $\mu$ :

$$
\mu^{n}\left(\gamma, \gamma^{\prime}\right)=\sum_{\gamma_{1} \in \Gamma} \ldots \sum_{\gamma_{n-1} \in \Gamma} \mu\left(\gamma, \gamma_{1}\right) \ldots \mu\left(\gamma_{n-1}, \gamma^{\prime}\right)
$$

We define the $n$-step energy $E_{\mu^{n}, \rho}(f)$ of a $\rho$-equivariant map $f$ by

$$
E_{\mu^{n}, \rho}(f)=\frac{1}{2} \sum_{\gamma \in \Gamma} \mu^{n}(e, \gamma) d_{Y}(f(e), f(\gamma))^{2} .
$$

2.1. Examples of $\boldsymbol{n}$-step energy. We first take a glance at examples of the computation of $E_{\mu^{n}, \rho}(f)$. In what follows, we drop $\rho$ in $E_{\mu^{n}, \rho}$ and use the symbol $E_{\mu^{n}}$, unless no confusion is likely to occur.

Example 1. Let $\Gamma=\mathbb{Z}$ and $\mu$ the standard random walk on $\mathbb{Z}$ :

$$
\mu(k, l)= \begin{cases}\frac{1}{2} & \text { if } k-l= \pm 1 \\ 0 & \text { otherwise }\end{cases}
$$

Let $Y=\mathbb{R}$ and $\rho: \mathbb{Z} \rightarrow \operatorname{Isom}(\mathbb{R})$ a homomorphism such that $\rho(1)(t)=u t+\tau$ for $t \in \mathbb{R}$, where $u= \pm 1$ and $\tau \in \mathbb{R}$. Let $f: \mathbb{Z} \rightarrow \mathbb{R}$ be a $\rho$-equivariant map such that $f(0)=\alpha \in \mathbb{R}$. Then for $k \in \mathbb{Z}$,

$$
\rho(k)(t)= \begin{cases}t+k \tau & \text { if } u=1, \\ t & \text { if } u=-1 \text { and } k \text { is even } \\ -t+\tau & \text { if } u=-1 \text { and } k \text { is odd }\end{cases}
$$

and

$$
f(k)= \begin{cases}\alpha+k \tau & \text { if } u=1 \\ \alpha & \text { if } u=-1 \text { and } k \text { is even, } \\ -\alpha+\tau & \text { if } u=-1 \text { and } k \text { is odd }\end{cases}
$$


Note that $\rho(\mathbb{Z})$ has a global fixed point in $\mathbb{R}$ exactly when $u=1$ and $\tau=0$, or $u=-1$, and $f$ is harmonic exactly when $u=1$, or $u=-1$ and $\alpha=\tau / 2$.

We now compute the $n$-step energy of $f$. Suppose that, among $n$ steps, a walker makes exactly $j$ steps to the right $(+1)$. Then the walker should make $n-j$ steps to the left $(-1)$, and he arrives at $2 j-n \in \mathbb{Z}$. There are ${ }_{n} C_{j}$ ways of such walks, each taking place with probability $(1 / 2)^{n}$. Therefore,

$$
\begin{aligned}
E_{\mu^{n}}(f) & =\frac{1}{2} \sum_{k \in \mathbb{Z}} \mu^{n}(0, k)|f(k)-f(0)|^{2} \\
& =\frac{1}{2} \sum_{j=0}^{n} \frac{{ }_{n} C_{j}}{2^{n}}|f(2 j-n)-f(0)|^{2} \\
& = \begin{cases}\frac{1}{2} \sum_{j=0}^{n} \frac{n C_{j}}{2^{n}}(2 j-n)^{2} \tau^{2}=\frac{n \tau^{2}}{2} & \text { if } u=1, \\
0 & \text { if } u=-1 \text { and } n \text { is even, } \\
2\left(\alpha-\frac{\tau}{2}\right)^{2} & \text { if } u=-1 \text { and } n \text { is odd. }\end{cases}
\end{aligned}
$$

We conclude that $E_{\mu^{n}}(f)=n E_{\mu}(f)$ for all $n$ if $u=1$, and $E_{\mu^{n}}(f) \leq E_{\mu}(f)$ for all $n$ if $u=-1$. In the computation above for the $u=1$ case, we have used the fact that

$$
\begin{gathered}
\sum_{j=0}^{n} \frac{{ }_{n} C_{j}}{2^{n}} j=(\text { the average of } B(n, 1 / 2))=\frac{n}{2}, \\
\sum_{j=0}^{n} \frac{{ }_{n} C_{j}}{2^{n}}\left(j-\frac{n}{2}\right)^{2}=(\text { the variance of } B(n, 1 / 2))=\frac{n}{4},
\end{gathered}
$$

where $B(n, 1 / 2)$ denotes the symmetric binomial distribution.

As we will see in $\S 2.3$ (Corollary 2.11), when the target space $Y$ is a Hilbert space, $E_{\mu^{n}}(f) \leq n E_{\mu}(f)$ holds for any $\rho$-equivariant map $f$, and the equality holds if and only if $f$ is harmonic.

Example 2. We take $\Gamma=F_{m}$ to be the free group of rank $m$ generated by $s_{1}, \ldots, s_{m}$. Let $S=\left\{s_{1}^{ \pm}, \ldots, s_{m}^{ \pm}\right\}$, and $\mu$ the standard random walk on $F_{m}$ with respect to the generator set $S$ :

$$
\mu\left(\gamma, \gamma^{\prime}\right)= \begin{cases}\frac{1}{2 m} & \text { if } \gamma^{\prime}=\gamma \text { sfor some } s \in S, \\ 0 & \text { otherwise. }\end{cases}
$$

Clearly, $\mu$ is $F_{m}$-invariant, finitely supported, symmetric, and irreducible. Let $Y$ be the Cayley graph of $F_{m}$ with respect to $S$. Then $Y$ is a $2 m$-regular tree. We give a distance on $Y$ by setting the length of each edge to be 1 . Let $\rho: F_{m} \rightarrow \operatorname{Isom}(Y)$ be the homomorphism that gives the action on $Y$ coming from the left action of $F_{m}$ 
on $F_{m}$ itself, and $f: F_{m} \rightarrow Y$ the standard embedding of $F_{m}$ into its Cayley graph $Y$. We give an estimate of $E_{\mu^{n}}(f)$. We denote by $\mu^{n}(r)$ the probability of a walker on $F_{m}$ being at distance $r$ from the starting point $e$ after taking $n$ steps following $\mu$. Thus

$$
E_{\mu^{n}}(f)=\frac{1}{2} \sum_{r=0}^{n} \mu^{n}(r) r^{2} .
$$

Let $X_{n}$ be the Bernoulli walk on $\mathbb{Z}$ starting from 0 which moves right with probability $p=(2 m-1) / 2 m$ and left with probability $q=1 / 2 m$. Denote by $b^{n}(r)$ the probability that $X_{n}=r \in \mathbb{Z}$ :

$$
b^{n}(r)={ }_{n} C_{(n+r) / 2}\left(\frac{2 m-1}{2 m}\right)^{(n+r) / 2}\left(\frac{1}{2 m}\right)^{(n-r) / 2}
$$

Note that the average $\mathbb{E}\left(X_{n}\right)$ and the variance $\mathbb{V}\left(X_{n}\right)$ are given by

$$
\mathbb{E}\left(X_{n}\right)=n(p-q)=n(m-1) / m \quad \text { and } \quad \mathbb{V}\left(X_{n}\right)=4 n p q=n(2 m-1) / m^{2},
$$

respectively. Recall that $\mu^{n}(r) \leq b^{n}(r) / p=2 m b^{n}(r) /(2 m-1)$ holds as explained in [21]. Then, by the variance equality,

$$
\begin{aligned}
E_{\mu^{n}}(f) & \leq \frac{1}{2} \sum_{r=0}^{n} \frac{2 m}{2 m-1} b^{n}(r) r^{2}=\frac{m}{2 m-1} \mathbb{E}\left(X_{n}^{2}\right) \\
& =\frac{m}{2 m-1}\left(\mathbb{V}\left(X_{n}\right)+\mathbb{E}\left(X_{n}\right)^{2}\right) \leq \frac{m}{2 m-1} n^{2} .
\end{aligned}
$$

2.2. General case. First we recall the well-known variance inequalities on a CAT(0) space.

Lemma 2.2. Let $Y$ be a CAT(0) space with metric $d$. Let $v=\sum_{i=1}^{m} t_{i} \operatorname{Dirac}_{v_{i}}$ be a probability measure with finite support on $Y$ and $\bar{v} \in Y$ the barycenter of $v$. Then we have

$$
\sum_{i=1}^{m} t_{i} d\left(v_{i}, w\right)^{2} \geq \sum_{i=1}^{m} t_{i} d\left(v_{i}, \bar{v}\right)^{2}+d(\bar{v}, w)^{2}
$$

for all $w \in Y$, and

$$
\frac{1}{2} \sum_{i=1}^{m} \sum_{j=1}^{m} t_{i} t_{j} d\left(v_{i}, v_{j}\right)^{2} \geq \sum_{i=1}^{m} t_{i} d\left(v_{i}, \bar{v}\right)^{2}
$$

Proof. We include a proof for the sake of completeness. Set $l=d(\bar{v}, w)$ and $F(w)=$ $\sum_{i=1}^{m} t_{i} d\left(v_{i}, w\right)^{2}$. Let $c:[0,1] \rightarrow Y$ be the (constant-speed) geodesic joining $\bar{v}$ and $w ; c(0)=\bar{v}, c(1)=w$. By Proposition 1.1, $d\left(v_{i}, c(\tau)\right)^{2}-(l \tau)^{2}$ is a convex function 
of $\tau$, and hence the same is true of the function $\varphi(\tau)=F(c(\tau))-(l \tau)^{2}$. Therefore,

$$
\begin{aligned}
F(\bar{v})-(l \tau)^{2} & \leq \varphi(\tau) \\
& \leq(1-\tau) \varphi(0)+\tau \varphi(1) \\
& =(1-\tau) F(\bar{v})+\tau\left(F(w)-l^{2}\right),
\end{aligned}
$$

and so $\tau F(\bar{v})+l^{2} \tau(1-\tau) \leq \tau F(w)$. Dividing the both sides by $\tau$ and letting $\tau \rightarrow 0$, we obtain (2.2). (2.3) follows by integrating (2.2) against $d v(w)$.

We use this lemma to derive the following

Lemma 2.3. Let $Y$ be a CAT(0) space and $p \in Y$. Let $v=\sum_{i=1}^{m} t_{i} \operatorname{Dirac}_{v_{i}}$ be a probability measure with finite support on $T C_{p} Y$ and $\bar{v} \in T C_{p} Y$ the barycenter of $v$. Then for any $w \in T C_{p} Y$, we have

$$
\langle\bar{v}, w\rangle \geq \sum_{i=1}^{m} t_{i}\left\langle v_{i}, w\right\rangle
$$

The equality holds if $w=\bar{v}$.

Proof. First we treat the $w=\bar{v}$ case (by just revising the proof of Lemma 2.7 in [11]). Set $\psi(\tau)=\sum_{i=1}^{m} t_{i} d\left(v_{i}, \tau \bar{v}\right)^{2}$, which takes its minimum at $\tau=1$. On the other hand, we can rewrite

$$
\psi(\tau)=\sum_{i=1}^{m} t_{i}\left|v_{i}\right|^{2}+\tau^{2}|\bar{v}|^{2}-2 \tau \sum_{i=1}^{m} t_{i}\left\langle v_{i}, \bar{v}\right\rangle,
$$

and the right-hand side takes its minimum at $\tau=\sum_{i=1}^{m} t_{i}\left\langle v_{i}, \bar{v}\right\rangle /|\bar{v}|^{2}$ (if $\bar{v} \neq 0_{p}$, which we may assume). Therefore,

$$
|\bar{v}|^{2}=\sum_{i=1}^{m} t_{i}\left\langle v_{i}, \bar{v}\right\rangle
$$

Now for $w$ arbitrary, applying (2.2) to $T C_{p} Y$ and rewriting in terms of the inner product on $T C_{p} Y$ and using (2.5), we obtain

$$
\sum_{i=1}^{m} t_{i}\left|v_{i}\right|^{2}+|w|^{2}-2 \sum_{i=1}^{m} t_{i}\left\langle v_{i}, w\right\rangle \geq \sum_{i=1}^{m} t_{i}\left|v_{i}\right|^{2}+|w|^{2}-2\langle\bar{v}, w\rangle .
$$

Cancelling out the common expression on the both sides, we obtain (2.4).

We restate Lemma 2.3 in the form which we will use later. 
Lemma 2.4. Let $\Gamma$ be a finitely generated group equipped with a $\Gamma$-invariant, finitely supported and symmetric random walk $\mu$, and $Y$ a CAT(0) space. Suppose that a homomorphism $\rho: \Gamma \rightarrow \operatorname{Isom}(Y)$ is given, and let $f: \Gamma \rightarrow Y$ be a $\rho$-equivariant map. Then for any $v \in T C_{f(e)} Y$,

$$
\left\langle-\Delta_{\mu} f(e), v\right\rangle \geq \sum_{\gamma \in \Gamma} \mu(e, \gamma)\left\langle F_{e}(\gamma), v\right\rangle
$$

holds.

We now prove the following

Proposition 2.5. Let $\mu, \mu^{\prime}$ be $\Gamma$-invariant, finitely supported and symmetric random walks on $\Gamma$. Then, for any $\rho$-equivariant map $f$,

$$
E_{\mu * \mu^{\prime}}(f) \geq E_{\mu}(f)+E_{\mu^{\prime}}(f)-\left\langle-\Delta_{\mu} f(e),-\Delta_{\mu^{\prime}} f(e)\right\rangle
$$

holds, where $\mu * \mu^{\prime}$ denotes the convolution of $\mu$ and $\mu^{\prime}$.

Proof. Since $\pi_{f(\gamma)}: Y \rightarrow T C_{f(\gamma)} Y$ is distance-nonincreasing, we obtain

$$
\begin{aligned}
E_{\mu * \mu^{\prime}}(f)= & \frac{1}{2} \sum_{\gamma, \gamma^{\prime}} \mu^{\prime}(e, \gamma) \mu\left(\gamma, \gamma^{\prime}\right) d\left(f(e), f\left(\gamma^{\prime}\right)\right)^{2} \\
\geq & \frac{1}{2} \sum_{\gamma, \gamma^{\prime}} \mu^{\prime}(e, \gamma) \mu\left(\gamma, \gamma^{\prime}\right) d_{f(\gamma)}\left(F_{\gamma}(e), F_{\gamma}\left(\gamma^{\prime}\right)\right)^{2} \\
= & \frac{1}{2} \sum_{\gamma, \gamma^{\prime}} \mu^{\prime}(e, \gamma) \mu\left(\gamma, \gamma^{\prime}\right)\left(\left|F_{\gamma}(e)\right|^{2}+\left|F_{\gamma}\left(\gamma^{\prime}\right)\right|^{2}-2\left\langle F_{\gamma}(e), F_{\gamma}\left(\gamma^{\prime}\right)\right\rangle\right) \\
= & \frac{1}{2} \sum_{\gamma} \mu^{\prime}(e, \gamma) d(f(e), f(\gamma))^{2}+\frac{1}{2} \sum_{\gamma} \mu^{\prime}(e, \gamma) \sum_{\gamma^{\prime}} \mu\left(e, \gamma^{-1} \gamma^{\prime}\right) \\
& \cdot d\left(f(e), f\left(\gamma^{-1} \gamma^{\prime}\right)\right)^{2}-\sum_{\gamma, \gamma^{\prime}} \mu^{\prime}(e, \gamma) \mu\left(\gamma, \gamma^{\prime}\right)\left\langle F_{\gamma}(e), F_{\gamma}\left(\gamma^{\prime}\right)\right\rangle,
\end{aligned}
$$

where we have used $\left|F_{\gamma}\left(\gamma^{\prime}\right)\right|=d\left(f(\gamma), f\left(\gamma^{\prime}\right)\right)=d\left(f(e), f\left(\gamma^{-1} \gamma^{\prime}\right)\right)$ and $\mu\left(\gamma, \gamma^{\prime}\right)=$ $\mu\left(e, \gamma^{-1} \gamma^{\prime}\right)$; these follow from the $\rho$-equivariance of $f$ and the $\Gamma$-invariance of $\mu$ respectively. The first and second terms in the last expression of (2.8) equal to $E_{\mu^{\prime}}(f)$ and $E_{\mu}(f)$ respectively. On the other hand, using Lemma 2.4 twice, we estimate the third term from below as

$$
\begin{aligned}
-\sum_{\gamma} \mu^{\prime}(e, \gamma) \sum_{\gamma^{\prime}} \mu\left(\gamma, \gamma^{\prime}\right)\left\langle F_{\gamma}(e), F_{\gamma}\left(\gamma^{\prime}\right)\right\rangle \\
\geq-\sum_{\gamma} \mu^{\prime}(e, \gamma)\left\langle F_{\gamma}(e),-\Delta_{\mu} f(\gamma)\right\rangle \\
\quad=-\sum_{\gamma} \mu^{\prime}\left(e, \gamma^{-1}\right)\left\langle F_{e}\left(\gamma^{-1}\right),-\Delta_{\mu} f(e)\right\rangle \\
\geq-\left\langle-\Delta_{\mu^{\prime}} f(e),-\Delta_{\mu} f(e)\right\rangle .
\end{aligned}
$$


To deduce the equality on the third line, one has to notice that $\rho\left(\gamma^{-1}\right)$ induces an isometry $\rho\left(\gamma^{-1}\right)_{*}: T C_{f(\gamma)} Y \rightarrow T C_{f(e)} Y$, which maps $F_{\gamma}(e)$ and $-\Delta_{\mu} f(\gamma)$ to $F_{e}\left(\gamma^{-1}\right)$ and $-\Delta_{\mu} f(e)$ respectively. We have also used $\mu^{\prime}(e, \gamma)=\mu^{\prime}\left(e, \gamma^{-1}\right)$. Combining these inequalities completes the proof.

Remark 1. Note that the difference between the both sides of (2.7) comes from the curvature of $Y$ and the nonlinearity of the tangent cones of $Y$. The former possibly makes the projection $Y \rightarrow T C_{p} Y$ distance-decreasing and causes strict inequality in (2.8). On the other hand, the latter may force the inequalities in (2.6), and thus in (2.9), to become strict ones. In particular, (2.7) becomes an equality when $Y$ is a Hilbert space.

Corollary 2.6. For any $\Gamma$-invariant, finitely supported and symmetric random walk $\mu, \rho$-equivariant map $f$, and positive integer $n$,

$$
E_{\mu^{n}}(f) \geq n E_{\mu}(f)-\sum_{i=1}^{n-1}\left\langle-\Delta_{i} f(e),-\Delta_{1} f(e)\right\rangle
$$

holds, where $-\Delta_{i} f(e)$ denotes the barycenter of $\left(F_{e}\right)_{*}\left(\mu^{i}(e, \cdot)\right)$.

Proof. To prove by induction, suppose that the inequality is true for $n-1$. Then by the proposition above

$$
\begin{aligned}
E_{\mu^{n}}(f) \geq & E_{\mu^{n-1}}(f)+E(f)-\left\langle-\Delta_{n-1} f(e),-\Delta_{1} f(e)\right\rangle \\
\geq & (n-1) E_{\mu}(f)-\sum_{i=1}^{n-2}\left\langle-\Delta_{i} f(e),-\Delta_{1} f(e)\right\rangle+E_{\mu}(f) \\
& -\left\langle-\Delta_{n-1} f(e),-\Delta_{1} f(e)\right\rangle \\
= & n E_{\mu}(f)-\sum_{i=1}^{n-1}\left\langle-\Delta_{i} f(e),-\Delta_{1} f(e)\right\rangle .
\end{aligned}
$$

This completes the proof of Corollary 2.6.

Remark 2. By the previous remark, (2.10) becomes an equality when $Y$ is a Hilbert space. See the next subsection for more on the Hilbertian case.

Remark 3. If $f$ is harmonic, then we have $E_{\mu^{n}}(f) \geq n E_{\mu}(f)$, and the strict inequality possibly holds by the reason as explained in Remark 1 . It is natural to expect that $E_{\mu^{n}}(f) / E_{\mu}(f)$ is bounded by a constant depending on some kind of growth rate of $Y$. As the following lemma shows, such a constant should not exceed $n^{2}$.

Lemma 2.7. Let $\mu$ be a $\Gamma$-invariant, finitely supported and symmetric random walk on $\Gamma$, and $f: \Gamma \rightarrow Y$ a $\rho$-equivariant map. Then the following estimates hold: 
(1) $\left|-\Delta_{\mu} f(e)\right|^{2} \leq 2 E_{\mu}(f)$.

(2) $E_{\mu^{n}}(f) \leq n^{2} E_{\mu}(f)$.

(3) $\left|-\Delta_{\mu^{n}} f(e)\right|^{2} \leq 2 n^{2} E_{\mu}(f)$.

Proof. We first prove (1). Using the variance inequality (2.2), we obtain

$$
\begin{aligned}
\left|-\Delta_{\mu} f(e)\right|^{2} & =d_{T C_{f(e)} Y}\left(0_{f(e)},-\Delta_{\mu} f(e)\right)^{2} \\
& \leq \sum_{\gamma \in \Gamma} \mu(e, \gamma) d_{T C_{f(e)} Y}\left(0_{f(e)}, F_{e}(\gamma)\right)^{2} \\
& =\sum_{\gamma \in \Gamma} \mu(e, \gamma) d_{Y}(f(e), f(\gamma))^{2}=2 E_{\mu}(f) .
\end{aligned}
$$

To prove (2), we compute

$$
\begin{aligned}
E_{\mu^{n}}(f) & =\frac{1}{2} \sum_{\gamma_{1}, \ldots, \gamma_{n}} \mu\left(e, \gamma_{1}\right) \cdots \mu\left(\gamma_{n-1}, \gamma_{n}\right) d\left(f(e), f\left(\gamma_{n}\right)\right)^{2} \\
& \leq \frac{1}{2} \sum_{\gamma_{1}, \ldots, \gamma_{n}} \mu\left(e, \gamma_{1}\right) \cdots \mu\left(\gamma_{n-1}, \gamma_{n}\right)\left(\sum_{i=1}^{n} d\left(f\left(\gamma_{i-1}\right), f\left(\gamma_{i}\right)\right)\right)^{2} \\
& \leq \frac{1}{2} \sum_{\gamma_{1}, \ldots, \gamma_{n}} \mu\left(e, \gamma_{1}\right) \cdots \mu\left(\gamma_{n-1}, \gamma_{n}\right) \cdot n \sum_{i=1}^{n} d\left(f\left(\gamma_{i-1}\right), f\left(\gamma_{i}\right)\right)^{2},
\end{aligned}
$$

where $\gamma_{0}=e$. Note that by $\Gamma$-invariance of $\mu$ and the $\rho$-equivariance of $f$,

$$
\begin{aligned}
& \frac{1}{2} \sum_{\gamma_{1}, \ldots, \gamma_{n}} \mu\left(e, \gamma_{1}\right) \ldots \mu\left(\gamma_{n-1}, \gamma_{n}\right) d\left(f\left(\gamma_{i-1}\right), f\left(\gamma_{i}\right)\right)^{2} \\
& \quad=\frac{1}{2} \sum_{\gamma_{i-1}, \gamma_{i}, \gamma_{n}} \mu^{i-1}\left(e, \gamma_{i-1}\right) \mu\left(\gamma_{i-1}, \gamma_{i}\right) \mu^{n-i}\left(\gamma_{i}, \gamma_{n}\right) d\left(f\left(\gamma_{i-1}\right), f\left(\gamma_{i}\right)\right)^{2} \\
& \quad=\frac{1}{2} \sum_{\gamma_{i-1}, \gamma_{i}} \mu^{i-1}\left(e, \gamma_{i-1}\right) \mu\left(\gamma_{i-1}, \gamma_{i}\right) d\left(f\left(\gamma_{i-1}\right), f\left(\gamma_{i}\right)\right)^{2} \\
& \quad=\frac{1}{2} \sum_{\gamma_{i-1}, \gamma_{i}} \mu^{i-1}\left(e, \gamma_{i-1}\right) \mu\left(e, \gamma_{i-1}^{-1} \gamma_{i}\right) d\left(f(e), f\left(\gamma_{i-1}^{-1} \gamma_{i}\right)\right)^{2} \\
& \quad=\sum_{\gamma_{i-1}} \mu^{i-1}\left(e, \gamma_{i-1}\right) E_{\mu}(f)=E_{\mu}(f) .
\end{aligned}
$$

Together with (2.11), this implies $E_{\mu^{n}}(f) \leq n^{2} E_{\mu}(f)$. Now (3) follows from (1) and (2).

Proposition 2.8. Let $\mu$ be a $\Gamma$-invariant, finitely supported, symmetric random walk and $f: \Gamma \rightarrow Y$ a $\rho$-equivariant map. Then

$$
E_{\mu^{n}}(f) \geq n E_{\mu}(f)-\frac{n(n-1)}{2} \sqrt{2 E_{\mu}(f)}\left|-\Delta_{\mu}(f)\right|
$$

holds. 
Proof. By Corollary 2.6 and Lemma 2.7 (3), we obtain

$$
\begin{aligned}
E_{\mu^{n}}(f) & \geq n E_{\mu}(f)-\sum_{i=1}^{n-1}\left\langle-\Delta_{i} f(e),-\Delta_{1} f(e)\right\rangle \\
& \geq n E_{\mu}(f)-\sum_{i=1}^{n-1} i \sqrt{2 E_{\mu}(f)}\left|-\Delta_{1} f(e)\right|
\end{aligned}
$$

This implies (2.12).

We can now prove

Theorem 2.9 (Gromov [8]). Suppose that there exist a positive integer $n$ and a positive real number $\varepsilon$ such that

$$
E_{\mu^{n}}(f) \leq(n-\varepsilon) E_{\mu}(f)
$$

holds for any $\rho$-equivariant map $f: \Gamma \rightarrow Y$. Then there exists a positive constant $C$ as in Proposition 2.1. In particular, $\rho(\Gamma)$ admits a global fixed point.

Proof. Suppose that (2.13) holds for a $\rho$-equivariant map $f$. By (2.12), we see

$$
(n-\varepsilon) E_{\mu}(f) \geq n E_{\mu}(f)-\frac{n(n-1)}{2} \sqrt{2 E_{\mu}(f)}\left|-\Delta_{\mu} f(e)\right|,
$$

from which we get

$$
\left|-\Delta_{\mu} f(e)\right|^{2} \geq \frac{2 \varepsilon^{2}}{n^{2}(n-1)^{2}} E_{\mu}(f) .
$$

This completes the proof of Theorem 2.9.

In the next section, we will need the following result, which is slightly more general than the above theorem and follows immediately from its proof.

Corollary 2.10. Suppose that there exist a positive integer $n$ and a positive real number $\varepsilon$ satisfying the following condition: for any $\rho$-equivariant map $f: \Gamma \rightarrow Y$, there exists $l \leq n$ such that

$$
E_{\mu l}(f) \leq(l-\varepsilon) E_{\mu}(f)
$$

Then there exists a positive constant $C$ as in Proposition 2.1. In particular, $\rho(\Gamma)$ admits a global fixed point. 
2.3. Affine case. Next we examine the behavior of $E_{\mu^{n}}(f)$ in the affine case, namely, the case when $Y$ is taken to be a real Hilbert space.

Let $\Gamma$ be a finitely generated group and $\mu$ a $\Gamma$-invariant, finitely supported and symmetric random walk on $\Gamma$. Let $\rho: \Gamma \rightarrow \operatorname{Isom}(\mathscr{H})$ be a homomorphism and $f: \Gamma \rightarrow \mathscr{H}$ a $\rho$-equivariant map, where $\mathscr{H}$ is a Hilbert space. Then $-\Delta_{\mu} f(\gamma)$ is given by

$$
-\Delta_{\mu} f(\gamma)=\sum_{\gamma^{\prime} \in \Gamma} \mu\left(\gamma, \gamma^{\prime}\right)\left(f\left(\gamma^{\prime}\right)-f(\gamma)\right)
$$

Recall that according to the semi-direct product decomposition $\operatorname{Isom}(\mathscr{H})=\mathcal{O}(\mathscr{H}) \ltimes$ $\mathscr{H}$, where $\mathcal{O}(\mathcal{H})$ is the orthogonal group of $\mathscr{H}, \rho$ is decomposed into the pair $\left(\rho_{0}, b\right)$ of a homomorphism $\rho_{0}: \Gamma \rightarrow \mathcal{O}(\mathcal{H})$ and a map $b: \Gamma \rightarrow \mathcal{H}$, so that $\rho(\gamma) v=$ $\rho_{0}(\gamma) v+b(\gamma)$ for $\gamma \in \Gamma$ and $v \in \mathscr{H}$. We note that $-\Delta_{\mu} f: \Gamma \rightarrow \mathcal{H}$ is $\rho_{0^{-}}$ equivariant; in fact,

$$
\begin{aligned}
-\Delta_{\mu} f\left(\gamma^{\prime} \gamma\right) & =\sum_{\gamma^{\prime \prime} \in \Gamma} \mu\left(\gamma^{\prime} \gamma, \gamma^{\prime \prime}\right)\left(f\left(\gamma^{\prime \prime}\right)-f\left(\gamma^{\prime} \gamma\right)\right) \\
& =\sum_{\gamma^{\prime \prime} \in \Gamma} \mu\left(\gamma, \gamma^{\prime-1} \gamma^{\prime \prime}\right)\left(\rho\left(\gamma^{\prime}\right) f\left(\gamma^{\prime-1} \gamma^{\prime \prime}\right)-\rho\left(\gamma^{\prime}\right) f(\gamma)\right) \\
& =\sum_{\gamma^{\prime \prime} \in \Gamma} \mu\left(\gamma, \gamma^{\prime-1} \gamma^{\prime \prime}\right) \rho_{0}\left(\gamma^{\prime}\right)\left(f\left(\gamma^{\prime-1} \gamma^{\prime \prime}\right)-f(\gamma)\right) \\
& =\rho_{0}\left(\gamma^{\prime}\right)\left(-\Delta_{\mu} f(\gamma)\right) .
\end{aligned}
$$

Note that we have used the $\Gamma$-invariance of $\mu$ and the linearity of $\rho_{0}\left(\gamma^{\prime}\right)$.

As in the general case, the set of $\rho_{0}$-equivariant maps from $\Gamma$ to $\mathscr{H}$, denoted by $\mathcal{M}_{\rho_{0}}$, is identified with $\mathscr{H}$ through the correspondence $\mathcal{M}_{\rho_{0}} \ni \varphi \mapsto \varphi(e) \in \mathscr{H}$. An inner product on $\mathcal{M}_{\rho_{0}}$ is defined in a natural way as

$$
\langle\varphi, \psi\rangle_{\mathcal{M}_{\rho_{0}}}:=\langle\varphi(e), \psi(e)\rangle(=\langle\varphi(\gamma), \psi(\gamma)\rangle) .
$$

We define an averaging operator $M$ by

$$
M \varphi(\gamma)=\sum_{\gamma^{\prime} \in \Gamma} \mu\left(\gamma, \gamma^{\prime}\right) \varphi\left(\gamma^{\prime}\right), \quad \varphi \in \mathcal{M}_{\rho_{0}} .
$$

Since $\rho_{0}(\gamma)$ is linear, we see that $M \varphi \in \mathcal{M}_{\rho_{0}}$ :

$$
\begin{aligned}
M \varphi\left(\gamma^{\prime \prime} \gamma\right) & =\sum_{\gamma^{\prime} \in \Gamma} \mu\left(\gamma^{\prime \prime} \gamma, \gamma^{\prime}\right) \varphi\left(\gamma^{\prime}\right) \\
& =\sum_{\gamma^{\prime} \in \Gamma} \mu\left(\gamma, \gamma^{\prime \prime-1} \gamma^{\prime}\right) \rho_{0}\left(\gamma^{\prime \prime}\right) \varphi\left(\gamma^{\prime \prime-1} \gamma^{\prime}\right) \\
& =\rho_{0}\left(\gamma^{\prime \prime}\right) M \varphi(\gamma) .
\end{aligned}
$$


Thus, $M$ is a linear operator acting on $\mathcal{M}_{\rho_{0}} \cong \mathscr{H}$. Since $\mu$ is symmetric and $\Gamma$ invariant, $M$ is selfadjoint:

$$
\begin{aligned}
\langle M \varphi, \psi\rangle_{\mathcal{M}_{\rho_{0}}} & =\langle M \varphi(e), \psi(e)\rangle=\sum_{\gamma} \mu(e, \gamma)\left\langle\rho_{0}(\gamma) \varphi(e), \psi(e)\right\rangle \\
& =\sum_{\gamma} \mu\left(e, \gamma^{-1}\right)\left\langle\varphi(e), \rho_{0}\left(\gamma^{-1}\right) \psi(e)\right\rangle \\
& =\langle\varphi, M \psi\rangle_{\mathcal{M}_{\rho_{0}}} .
\end{aligned}
$$

Using this operator $M$, we can rewrite $-\Delta_{n} f$, where $-\Delta_{n}=-\Delta_{\mu^{n}}$, as follows.

$$
\begin{aligned}
-\Delta_{n} f(\gamma) & =\sum_{\gamma^{\prime} \in \Gamma} \mu^{n}\left(\gamma, \gamma^{\prime}\right)\left(f\left(\gamma^{\prime}\right)-f(\gamma)\right) \\
& =\sum_{\gamma_{1}, \gamma^{\prime} \in \Gamma} \mu\left(\gamma, \gamma_{1}\right) \mu^{n-1}\left(\gamma_{1}, \gamma^{\prime}\right)\left(f\left(\gamma^{\prime}\right)-f\left(\gamma_{1}\right)+f\left(\gamma_{1}\right)-f(\gamma)\right) \\
& =\sum_{\gamma_{1}} \mu\left(\gamma, \gamma_{1}\right)\left(-\Delta_{n-1} f\left(\gamma_{1}\right)+f\left(\gamma_{1}\right)-f(\gamma)\right) \\
& =M\left(-\Delta_{n-1} f\right)(\gamma)+\left(-\Delta_{1} f\right)(\gamma),
\end{aligned}
$$

and thus,

$$
-\Delta_{n} f=M\left(-\Delta_{n-1} f\right)+\left(-\Delta_{1} f\right) .
$$

Proceeding inductively, we see that

$$
-\Delta_{n} f=\left(M^{n-1}+M^{n-2}+\cdots+M+I\right)\left(-\Delta_{1} f\right) .
$$

In particular, we see that if $f$ is $\mu$-harmonic, then $f$ must be $\mu^{n}$-harmonic.

Corollary 2.11. Let $Y$ be a Hilbert space. For any $\Gamma$-invariant, finitely supported and symmetric random walk $\mu$ on $\Gamma, \rho$-equivariant map $f: \Gamma \rightarrow Y$, and a positive integer $n$,

$$
E_{\mu^{n}}(f) \leq n E_{\mu}(f)
$$

holds. The equality holds if and only if $f$ is harmonic.

Proof. According to Corollary 2.6 and Remark 2, it is enough to show that $\left\langle-\Delta_{i} f(e),-\Delta_{1} f(e)\right\rangle \geq 0$ for each $i$ in order to prove the inequality. When $i=1$, this is obvious. Suppose that $i=2 m+2, m \geq 0$. By (2.14), we get

$$
\begin{aligned}
\left\langle-\Delta_{i} f(e),-\Delta_{1} f(e)\right\rangle & =\sum_{k=0}^{m}\left\langle\left(M^{2 k}+M^{2 k+1}\right)\left(-\Delta_{1} f\right)(e),-\Delta_{1} f(e)\right\rangle \\
& =\sum_{k=0}^{m}\left\langle(I+M) M^{k}\left(-\Delta_{1} f\right)(e), M^{k}\left(-\Delta_{1} f\right)(e)\right\rangle
\end{aligned}
$$


since $M$ is selfadjoint. Now for $\varphi \in \mathcal{M}_{\rho_{0}}$,

$$
\langle(I+M) \varphi, \varphi\rangle_{\mathcal{M}_{\rho_{0}}}=\langle\varphi(e), \varphi(e)\rangle+\sum_{\gamma \in \Gamma} \mu(e, \gamma)\left\langle\rho_{0}(\gamma) \varphi(e), \varphi(e)\right\rangle \geq 0,
$$

where we have used $\left|\left\langle\rho_{0}(\gamma) \varphi(e), \varphi(e)\right\rangle\right| \leq|\varphi(e)|^{2}$ which holds since $\rho_{0}(\gamma)$ is orthogonal. Thus the operator $I+M$ is nonnegative, and applying this to (2.15), we obtain $\left\langle-\Delta_{i} f(e),-\Delta_{1} f(e)\right\rangle \geq 0$.

Suppose that $i=2 m+3, m \geq 0$. Then (by (2.14) again)

$$
\begin{aligned}
& \left\langle-\Delta_{i} f(e),-\Delta_{1} f(e)\right\rangle \\
& \quad=\left\langle-\Delta_{i-1} f(e),-\Delta_{1} f(e)\right\rangle+\left\langle M^{m+1}\left(-\Delta_{1} f(e)\right), M^{m+1}\left(-\Delta_{1} f(e)\right)\right\rangle \geq 0 .
\end{aligned}
$$

Now suppose that $E_{\mu^{n}}(f)=n E_{\mu}(f)$. Then $\left\langle-\Delta_{1} f(e),-\Delta_{1} f(e)\right\rangle=0$, and hence $f$ is harmonic. The converse is obvious.

2.4. Converse of Theorem 2.9. The following proposition shows that an assertion slightly stronger than the converse of Theorem 2.9 holds for some CAT(0) spaces.

Proposition 2.12. Let $Y$ be either a CAT(0) Riemannian manifold or an $\mathbb{R}$-tree, and $\rho: \Gamma \rightarrow \operatorname{Isom}(Y)$ a homomorphism. Suppose that $\rho(\Gamma)$ admits a global fixed point. Then there exists a positive constant $C_{\rho}$ such that $E_{\mu^{n}}(f) \leq C_{\rho} E_{\mu}(f)$ for any $n \in \mathbb{N}$ and $\rho$-equivariant map $f$. In particular, taking $n>C_{\rho}$, we obtain $n, \varepsilon$ as in Theorem 2.9.

Proof. Let $f: \Gamma \rightarrow Y$ be a $\rho$-equivariant map. Denote by $F$ the fixed-point set of $\rho(\Gamma)$, and let $p_{0} \in F$ be the nearest point from $f(e)$. Since $f(e) \in F$ implies $E_{\mu}(f)=E_{\mu^{n}}(f)=0$, we may assume $f(e) \notin F$. Set $R=d\left(f(e), p_{0}\right)=$ $d(f(e), F)$. Since, for any $\gamma \in \Gamma, d\left(f(\gamma), p_{0}\right)=R$, and hence $d(f(e), f(\gamma)) \leq$ $2 R$, we see that $E_{\mu^{n}}(f) \leq 2 R^{2}$ for any $n \in \mathbb{N}$. Let $S=\{s \in \Gamma \mid \mu(e, s) \neq 0\}$. Suppose that there exists a positive constant $\kappa$ such that $\max \left\{L_{p_{0}}(f(e), f(s)) \mid s \in\right.$ $S\} \geq \kappa$ holds for any $\rho$-equivariant map $f$. Then, for any $\rho$-equivariant map $f$, we have $\max \{d(f(e), f(s)) \mid s \in S\} \geq 2 R \sin (\kappa / 2)$. This implies that

$$
E_{\mu}(f) \geq 2 R^{2} \sin ^{2} \frac{\kappa}{2} \min _{s \in S} \mu(e, s),
$$

and we can take

$$
C_{\rho}=\left(\sin ^{2} \frac{\kappa}{2} \min _{s \in S} \mu(e, s)\right)^{-1} .
$$

Suppose that $Y$ is a Riemannian manifold. Note that $f(e)$ lies in a geodesic starting from $p_{0}$ which is normal to $F$, and $p_{0}$ depends on $f$. So take any point $p \in F$, and set

$$
\kappa_{p}=\inf _{V \in T_{p} F^{\perp},|V|=1} \max \left\{L_{p}\left(V, \rho(s)_{*} V\right) \mid s \in S\right\},
$$


where $\rho(s)_{*}$ denotes the differential of $\rho(s)$, which induces an isometry on $T_{p} F^{\perp}$. Note that $\kappa_{p}$ is positive. In fact, since $T_{p} F^{\perp}$ is finite-dimensional, $\kappa_{p}=0$ implies the existence of a unit vector $V \in T_{p} F^{\perp}$ fixed by $\rho(s)_{*}$ for any $s \in S$, and hence fixed by $\rho(\gamma)_{*}$ for any $\gamma \in \Gamma$ since $S$ generates $\Gamma$ by the $\Gamma$-invariance and the irreducibility of $\mu$. Then the geodesic $\exp t V$ must be fixed by $\rho(\Gamma)$. This contradicts the definition of $F$, since $V$ is normal to $F$. Let $q \in F$ be another point in $F$. Let $c:[0,1] \rightarrow Y$ be the unique geodesic starting from $p$ and terminating at $q$, and $P_{t}: T_{p} Y \rightarrow T_{c(t)} Y$ the parallel translation along $c$. Note that $c$ must lie in $F$, and hence it is fixed by $\rho(\Gamma)$. Therefore, for any $V \in T_{p} V, t \mapsto \rho(s)_{*} P_{t}(V)$ is a parallel vector field along $c$ with initial vector $\rho(s)_{*} P_{0}(V)=\rho(s)_{*} V$. By the uniqueness of a parallel vector field with a given initial condition, we see that $\rho(s)_{*} P_{1}(V)=P_{1}\left(\rho(s)_{*} V\right)$, namely $\rho(s)_{*}$ commutes with $P_{1}$. Thus the action of $\rho(s)$ on $T_{q} Y$ is conjugate to that on $T_{p} Y$ by $P_{1}$. In particular, $\kappa_{p}=\kappa_{q}$, that is, $\kappa_{p}$ does not depend on the choice of $p \in F$. Hence we can take $\kappa$ above to be $\kappa_{p}$.

Now suppose that $Y$ is an $\mathbb{R}$-tree, and let $f, F, p_{0}$ and $R$ be as above. Since $Y$ is an $\mathbb{R}$-tree, the angle $\angle_{p_{0}}(f(e), f(s))$ equals either 0 or $\pi$. Suppose that there exists $s \in S$ such that $\left[p_{0}, f(e)\right] \cap\left[p_{0}, f(s)\right]=\left\{p_{0}\right\}$, where $\left[p_{0}, q\right]$ denotes the geodesic segment joining $p_{0}$ and $q$. Then $\left[p_{0}, f(e)\right] \cup\left[p_{0}, f(s)\right]$ is an arc (a topological segment) joining $f(e)$ and $f(s)$, which must be unique in $Y$ by the definition of $\mathbb{R}$-tree. In other words, $\left[p_{0}, f(e)\right] \cup\left[p_{0}, f(s)\right]$ is a geodesic segment joining $f(e)$ and $f(s)$. Therefore $\angle_{p_{0}}(f(e), f(s))=\pi$. Now assume the contrary: $\left[p_{0}, f(e)\right] \cap\left[p_{0}, f(s)\right] \neq\left\{p_{0}\right\}$ for all $s \in S$. Let $c_{s}:[0, R] \rightarrow Y, s \in S$, and $c_{e}:[0, R] \rightarrow Y$ be unit speed geodesics starting from $p_{0}$ and terminating at $f(s)$ and $f(e)$ respectively. By our assumption, there exists a positive constant $T_{s}$ for each $s \in S$ such that $c_{s}\left(\left[0, T_{s}\right]\right) \subset c_{e}([0, R])$. Since the geodesics are of unit speed, this means $\left.c_{s}\right|_{\left[0, T_{S}\right]}=\left.c_{e}\right|_{\left[0, T_{S}\right]}$ for each $s \in S$. Because $S$ is a finite set, we get a positive constant $T:=\min \left\{T_{s} \mid s \in S\right\}$. By the definition of $T,\left.c_{e}\right|_{[0, T]}=\left.c_{s}\right|_{[0, T]}$ for all $s \in S$ and $c_{e}([0, T]) \neq\left\{p_{0}\right\}$. It is clear that $c_{e}([0, T])$ must be fixed by $\rho(s)$ for all $s \in S$, and hence by $\rho(\Gamma)$. This means that there is a fixed point $p=c(T)$ of $\rho(\Gamma)$ which is closer to $f(e)$ than $p_{0}$. This contradicts our choice of $p_{0}$. Therefore, for any $\rho$-equivariant map $f$, $\max \left\{L_{p_{0}}(f(e), f(s)) \mid s \in S\right\}$ must be equal to $\pi$, and we can take $\kappa$ to be $\pi$. This completes the proof.

Remark 4. From the proof, one sees that $C_{\rho}$ for an $\mathbb{R}$-tree equals $\left(\min _{s \in S} \mu(e, s)\right)^{-1}$ and does not depend on $\rho$. It is plausible that Proposition 2.12 is also true for Euclidean buildings.

\section{Fixed-point property of random groups}

In this section, we will prove that a random group of Gromov's graph model associated with a sequence of expanders satisfying some additional conditions has the fixed-point property for a certain large class of $\mathrm{CAT}(0)$ spaces. 
3.1. Preliminaries on graphs. Let $G=(V, E)$ be a finite connected graph, where $V$ and $E$ are the sets of vertices and undirected edges, respectively. We denote the set of directed edges by $\vec{E}$. Let $\mu_{G}$ and $\nu_{G}$ denote the standard random walk on $G$ and the standard probability measure on $V$ given by

$$
\mu_{G}(u, v)=\left\{\begin{array}{ll}
\frac{1}{\operatorname{deg}(u)} & \text { if }\{u, v\} \in E, \\
0 & \text { otherwise, }
\end{array} \quad \text { and } \quad v_{G}(u)=\frac{\operatorname{deg}(u)}{2|E|}\right.
$$

respectively. Note that $\mu_{G}$ is symmetric with respect to $v_{G}: v_{G}(u) \mu_{G}(u, v)=$ $v_{G}(v) \mu_{G}(v, u)$. The discrete Laplacian $\Delta_{G}$ of $G$, acting on real-valued functions $\varphi$ on $V$, is defined by

$$
\left(\Delta_{G} \varphi\right)(u)=\varphi(u)-\sum_{v \in V} \mu(u, v) \varphi(v), \quad u \in V .
$$

Let $\lambda_{1}(G, \mathbb{R})$ denote the second eigenvalue of $\Delta_{G}$. It is characterized variationally as

$$
\lambda_{1}(G, \mathbb{R})=\inf _{\varphi} \frac{\frac{1}{2} \sum_{u \in V} v_{G}(u) \sum_{v \in V} \mu_{G}(u, v)(\varphi(u)-\varphi(v))^{2}}{\sum_{u \in V} v_{G}(u)(\varphi(u)-\bar{\varphi})^{2}},
$$

where $\varphi$ is a nonconstant real-valued function on $V$, and $\bar{\varphi}$ denotes the average of $\varphi$, given by $\bar{\varphi}=\left[\sum_{u \in V} \operatorname{deg}(u) \varphi(u)\right] /\left[\sum_{u \in V} \operatorname{deg}(u)\right]$. The girth of $G$, denoted by $\operatorname{girth}(G)$, is the minimal length of a cycle (i.e., a closed path) in $G$, and the diameter of $G$, denoted by $\operatorname{diam}(G)$, is the maximum distance between a pair of points in $G$.

Let $\left\{G_{l}=\left(V_{l}, E_{l}\right)\right\}_{l \in L}$ be a sequence of finite connected graphs with $L$ an unbounded set of positive integers and $\left|V_{l}\right| \rightarrow \infty$ as $l \rightarrow \infty$. We say that $\left\{G_{l}\right\}_{l \in L}$ is a sequence of (bounded degree) expanders if it satisfies the following conditions for some positive integer $d_{0}$ and positive real number $\mu_{0}$ :

(i) $2 \leq \operatorname{deg}(u) \leq d_{0}$ for all $l \in L$ and all $u \in V_{l}$,

(ii) $\lambda_{1}\left(G_{l}, \mathbb{R}\right) \geq \mu_{0}$ for all $l \in L$.

3.2. Graph-model random groups and their hyperbolicity. We first recall the formulation of Gromov's graph model of random groups [8], [20]. Let $\Gamma=F_{k}$ be the free group generated by $S=\left\{s_{1}^{ \pm}, \ldots, s_{k}^{ \pm}\right\}$. Let $G=(V, E)$ be a finite connected graph, and we use the notations as in the previous subsection. A map $\alpha: \vec{E} \rightarrow S$ satisfying $\alpha((u, v))=\alpha((v, u))^{-1}$ for all $(u, v) \in \vec{E}$ is called an $S$ labelling of $G$. For such an $\alpha$ and a path $\vec{p}=\left(\vec{e}_{1}, \ldots, \vec{e}_{l}\right)$ in $G$, where $\vec{e}_{i} \in \vec{E}$, define $\alpha(\vec{p})=\alpha\left(\vec{e}_{1}\right) \cdots \alpha\left(\vec{e}_{l}\right) \in \Gamma$. Then set $R_{\alpha}=\{\alpha(\vec{c}) \mid \vec{c}$ is a cycle in $G\}$ and $\Gamma_{\alpha}=\Gamma / \overline{R_{\alpha}}$, where $\overline{R_{\alpha}}$ is the normal closure of $R_{\alpha}$. Let $\Lambda(G, k)$ denote the set of all $S$-labellings of $G$, consisting of $(2 k)^{|E|}$ elements, and make it into a probability space by putting a uniform probability measure on it. When $|V| \rightarrow \infty$, the group $\Gamma_{\alpha}$ for a randomly and uniformly chosen $\alpha \in \Lambda(G, k)$ is a 'random group'.

To be precise, choose a sequence of finite connected graphs $\left\{G_{l}=\left(V_{l}, E_{l}\right)\right\}_{l \in L}$ with $L$ an unbounded set of positive integers and $\left|V_{l}\right| \rightarrow \infty$ as $l \rightarrow \infty$. Given a 
group property $\mathrm{P}$ (e.g., Kazhdan's property (T)), we say that a random group has property $P$ if the probability of $\Gamma_{\alpha}$ having property $\mathrm{P}$ goes to one as $l \rightarrow \infty$, that is, if $\mid\left\{\alpha \in \Lambda\left(G_{l}, k\right) \mid \Gamma_{\alpha}\right.$ has property $\left.\mathrm{P}\right\}|/| \Lambda\left(G_{l}, k\right) \mid \rightarrow 1$ as $l \rightarrow \infty$. In actual use, we primarily assume that $\left\{G_{l}\right\}_{l \in L}$ is a sequence of expanders. In what follows, we will make precise what kind of properties the expanders should further have in order to prove the corresponding graph model useful for our purpose.

We begin with the specific example of expanders that was discovered by Lubotzky, Phillips and Sarnak [16].

Example 3. Let $p$ and $q$ be distinct primes which are congruent to 1 modulo 4 . The LPS expanders $X^{p, q}$ are $(p+1)$-regular Cayley graphs of the group PSL $\left(2, \mathbb{F}_{q}\right)$ if the Legendre symbol $\left(\frac{p}{q}\right)=1$ and of $\operatorname{PGL}\left(2, \mathbb{F}_{q}\right)$ if $\left(\frac{p}{q}\right)=-1$, where $\mathbb{F}_{q}$ is a finite field with $q$ elements $(\cong \mathbb{Z} / q \mathbb{Z})$. They are so-called Ramanujan graphs and also satisfy some other extremal combinatorial properties:

Case i. $\left(\frac{p}{q}\right)=-1 ; X^{p, q}$ is bipartite of order $n=\left|X^{p, q}\right|=q\left(q^{2}-1\right)$,

(a) $\operatorname{girth}\left(X^{p, q}\right) \geq 4 \log _{p} q-\log _{p} 4$,

(b) $\operatorname{diam}\left(X^{p, q}\right) \leq 2 \log _{p} n+2 \log _{p} 2+1$.

Case ii. $\left(\frac{p}{q}\right)=1 ; n=\left|X^{p, q}\right|=q\left(q^{2}-1\right) / 2$ and $X^{p, q}$ is not bipartite,

(a) $\operatorname{girth}\left(X^{p, q}\right) \geq 2 \log _{p} q$,

(b) $\operatorname{diam}\left(X^{p, q}\right) \leq 2 \log _{p} n+2 \log _{p} 2+1$.

Let us introduce a new parameter $l=\left[\log _{p} q\right]$, where $p$ is fixed and $q$ varies, and set $G_{l}=X^{p, q}$. (Note that the map $q \mapsto l$ is not one-to-one. So for each $l$ we choose a single $q$ among those mapped to $l$.) Then in the both cases the conditions (a), (b) are rewritten as

$$
\operatorname{girth}\left(G_{l}\right) \geq \text { const }_{1} \cdot l \quad \text { and } \operatorname{diam}\left(G_{l}\right) \leq \text { const }_{2} \cdot l
$$

respectively. Note that one can choose const $_{1}=2$ and const $2=6+o_{l}(1)$.

With this example as a model, we consider a sequence of finite connected graphs $\left\{G_{l}=\left(V_{l}, E_{l}\right)\right\}_{l \in L}$ with $L$ an unbounded set of positive integers satisfying the following conditions for some positive integer $d_{0}$ and positive real number $\mu_{0}$ :

(i) $3 \leq \operatorname{deg}(u) \leq d_{0}$ for all $l \in L$ and all $u \in V_{l}$,

(ii) $\operatorname{girth}\left(G_{l}\right) \geq l$ and $\operatorname{diam}\left(G_{l}\right) \leq$ const $\cdot l$ for all $l \in L$,

(iii) $\lambda_{1}\left(G_{l}, \mathbb{R}\right) \geq \mu_{0}$ for all $l \in L$.

For a fixed positive integer $j$, we also consider the graph $G_{l}^{(j)}$ obtained from $G_{l}$ by subdividing every edge of $G_{l}$ into $j$ edges by adding $j-1$ vertices. Set $l^{\prime}=j l$ so that $l^{\prime}$ varies over $j L$. Then the sequence of graphs $\left\{G_{l^{\prime} / j}^{(j)}\right\}_{l^{\prime} \in j L}$ satisfies the following conditions:

(i') $2 \leq \operatorname{deg}(u) \leq d_{0}$ for all $l^{\prime} \in j L$ and all $u \in V\left(G_{l^{\prime} / j}^{(j)}\right)$, 
(ii') $\operatorname{girth}\left(G_{l^{\prime} / j}^{(j)}\right) \geq l^{\prime}$ and $\operatorname{diam}\left(G_{l^{\prime} / j}^{(j)}\right) \leq \mathrm{const} \cdot l^{\prime}$ for all $l^{\prime} \in j L$,

(iii') $\lambda_{1}\left(G_{l^{\prime} / j}^{(j)}, \mathbb{R}\right) \geq c\left(\mu_{0}, j\right)>0$ for all $l^{\prime} \in j L$.

(For (iii'), see [21].) Moreover, if an arbitrary $\beta>1$ is given, then by choosing $j$ large enough, we can arrange so that $\left\{G_{l^{\prime} / j}^{(j)}\right\}_{l^{\prime} \in j L}$ satisfies

$\left(\mathrm{iv}^{\prime}\right)$ the number of embedded paths in $G_{l^{\prime} / j}^{(j)}$ of length less than $\frac{l^{\prime}}{2}$ is less than const $\cdot \beta^{l^{\prime} / 2}$.

(For this point, we refer the reader to [5], p. 17.)

Henceforth, we will fix a sequence of finite connected graphs $\left\{G_{l}\right\}_{l \in L}$ satisfying the conditions (i)-(iii). We will also fix a sufficiently large $j$, and consider the graph model of random groups associated with the sequence of graphs $\left\{G_{l^{\prime} / j}^{(j)}\right\}_{l^{\prime} \in j L}$. The fact that a random group of this model is an infinite group follows from the following theorem due to Gromov [8] (see also [5]).

Theorem 3.1. Let $\left\{G_{l}=\left(V_{l}, E_{l}\right)\right\}_{l \in L}$ be a sequence of finite connected graphs with $L$ an unbounded set of positive integers. Suppose that $\left\{G_{l}\right\}_{l \in L}$ satisfies the following conditions for some positive integer $d_{0}$ and a choice of $\beta>1$ sufficiently close to 1 :

(i) $2 \leq \operatorname{deg}(u) \leq d_{0}$ for all $l \in L$ and all $u \in V_{l}$,

(ii) $\operatorname{girth}\left(G_{l}\right) \geq l$ and $\operatorname{diam}\left(G_{l}\right) \leq$ const $\cdot l$ for all $l \in L$,

(iii) the number of embedded paths in $G_{l}$ oflength less than $\frac{l}{2}$ is less than const. $\beta^{l / 2}$.

Then a random group of the graph model associated with $\left\{G_{l}\right\}_{l \in L}$ is non-elementary hyperbolic; in particular, it is an infinite group.

3.3. Fixed-point theorem. We first recall (see [22])

Definition 3.2. For a finite connected graph $G$ and a $\operatorname{CAT}(0)$ space $T$, the Wang invariant $\lambda_{1}(G, T)$ is defined by $\lambda_{1}(G, T)=\inf \operatorname{RQ}(\varphi)$, where the infimum is taken over all nonconstant maps $\varphi: V \rightarrow T$ and

$$
\operatorname{RQ}(\varphi)=\frac{\frac{1}{2} \sum_{u \in V} v_{G}(u) \sum_{v \in V} \mu_{G}(u, v) d_{T}(\varphi(u), \varphi(v))^{2}}{\sum_{u \in V} v_{G}(u) d_{T}\left(\varphi(u), \operatorname{bar}\left(\varphi_{*} v_{G}\right)\right)^{2}}
$$

Theorem 3.3. Given positive integers $k, d_{0}$ and positive real number $\lambda_{0}$, there exists $g_{0}=g_{0}\left(\lambda_{0}\right)$ such that if $G=(V, E)$ is a finite connected graph and $y$ is a family of CAT(0) spaces satisfying

(i) $2 \leq \operatorname{deg}(u) \leq d_{0}$ for all $u \in V$,

(ii) $\operatorname{girth}(G) \geq g_{0}$,

(iii) $\lambda_{1}\left(G, T C_{p} Y\right) \geq \lambda_{0}$ for all $Y \in \mathcal{Y}$ and all $p \in Y$,

then with probability at least $1-a_{1} e^{-a_{2}|V|}$, where $a_{1}=a_{1}\left(k, \lambda_{0}\right)$ and $a_{2}=$ $a_{2}\left(k, d_{0}, \lambda_{0}\right), \Gamma_{\alpha}$ has property $F Y$. 
The geometric part of the proof of the theorem is based on Corollary 2.10. We use it with the following setting: the group $\Gamma$ is the free group $F_{k}$ generated by $S=\left\{s_{1}^{ \pm}, \ldots, s_{k}^{ \pm}\right\}$, and the random walk $\mu$ is the standard one, that is, it is given by

$$
\mu\left(\gamma, \gamma^{\prime}\right)= \begin{cases}\frac{1}{2 k} & \text { if } \gamma^{\prime}=\gamma s \text { for some } s \in S, \\ 0 & \text { otherwise. }\end{cases}
$$

The probabilistic part of the proof of Theorem 3.3 is based on the following proposition. A similar proposition was formulated and proved by Silberman [21] when the target space is a Hilbert space, in the course of detailing Gromov's argument in [8], 3.12. Our proof is simpler than Silberman's, and we will present it in the Appendix. (Our proof, however, is less elementary than Silberman's, as we replace his explicit calculation of binomial coefficients by use of the central limit theorem.)

Proposition 3.4 (cf. [21], Proposition 2.14). Suppose that $G=(V, E)$ is a finite connected graph and $n$ is a positive integer satisfying

(i) $2 \leq \operatorname{deg}(u) \leq d$ for all $u \in V$,

(ii) $2 \leq n \leq \operatorname{girth}(G) / 2$.

Then with probability at least $1-a_{1} e^{-a_{2}|V|}, a_{1}=a_{1}(k, n), a_{2}=a_{2}(k, d, n)$, the following assertion holds: for any CAT(0) space $Y$, any homomorphism $\rho^{(\alpha)}: \Gamma_{\alpha} \rightarrow$ $\operatorname{Isom}(Y)$ and any $\rho^{(\alpha)}$-equivariant map $f^{(\alpha)}: \Gamma_{\alpha} \rightarrow Y$, there exists an $l$ (depending on $\left.f^{(\alpha)}\right), \sqrt{n}<l \leq n$, such that

$$
E_{\mu^{l}, \rho}(f) \leq \frac{C}{\lambda_{1}(G, Y)} E_{\mu, \rho}(f),
$$

where $\rho=\rho^{(\alpha)} \circ \mathrm{pr}, f=f^{(\alpha)} \circ \mathrm{pr}$ with $\mathrm{pr}$ denoting the projection from $\Gamma$ onto $\Gamma_{\alpha}$, and $C$ is an absolute constant.

Proof of Theorem 3.3. For any CAT(0) space $Y$, it is easy to verify that $\lambda_{1}(G, Y) \geq$ $\inf _{p \in Y} \lambda_{1}\left(G, T C_{p} Y\right)$ (see [22]). Therefore, for any $Y \in \mathcal{Y}$, we have $\lambda_{1}(G, Y) \geq \lambda_{0}$.

Now let $n$ be the minimum positive integer satisfying $C / \lambda_{0}<\sqrt{n}$, and set $g_{0}=2 n$. Then the assertion of Proposition 3.4 holds with the high probability as stated there. Therefore, if $Y \in \mathcal{Y}$, we obtain

$$
E_{\mu^{l}, \rho}(f) \leq \frac{C}{\lambda_{0}} E_{\mu, \rho}(f) \leq(l-\varepsilon) E_{\mu, \rho}(f),
$$

where $\varepsilon=\sqrt{n}-C / \lambda_{0}$. By Corollary 2.10, $\rho(\Gamma)=\rho^{(\alpha)}\left(\Gamma_{\alpha}\right)$ fixes a point in $Y$.

Combining Theorem 3.1 and Theorem 3.3, we obtain

Theorem 3.5. Let $\left\{G_{l}=\left(V_{l}, E_{l}\right)\right\}_{l \in L}$ be a sequence of finite connected graphs with $L$ an unbounded set of positive integers, and let $y$ be a family of CAT(0) spaces. Suppose that they satisfy the following conditions for some positive integer $d_{0}$, positive real number $\lambda_{0}$ and a choice of $\beta>1$ sufficiently close to 1 : 
(i) $2 \leq \operatorname{deg}(u) \leq d_{0}$ for all $l \in L$ and all $u \in V_{l}$,

(ii) $\operatorname{girth}\left(G_{l}\right) \geq l$ and $\operatorname{diam}\left(G_{l}\right) \leq$ const $\cdot l$ for all $l \in L$,

(iii) $\lambda_{1}\left(G_{l}, T C_{p} Y\right) \geq \lambda_{0}$ for all $l \in L$, all $Y \in \mathcal{Y}$ and all $p \in Y$,

(iv) the number of embedded paths in $G_{l}$ of length less than $\frac{l}{2}$ is less than const $\cdot \beta^{l / 2}$.

Then a random group of the graph model associated with $\left\{G_{l}\right\}_{l \in L}$ is infinite hyperbolic and has property $F Y$.

To formulate a class of CAT(0) spaces so that the condition (iii) of Theorem 3.5 is satisfied, we recall the definition of the invariant of a CAT(0) space introduced in [11].

Definition 3.6. Let $T$ be a CAT(0) space. Let $\mu=\sum_{i=1}^{m} t_{i} \operatorname{Dirac}_{v_{i}}$ be a probability measure with finite support on $T$ and $\bar{\mu} \in T$ the barycenter of $\mu$. Consider all maps $\iota: \operatorname{supp} \mu \rightarrow \mathbb{R}^{m}$ satisfying

$$
\left\|\iota\left(v_{i}\right)\right\|=d_{T}\left(\bar{\mu}, v_{i}\right), \quad\left\|\iota\left(v_{i}\right)-\iota\left(v_{j}\right)\right\| \leq d_{T}\left(v_{i}, v_{j}\right),
$$

and set

$$
\delta(\mu)=\inf _{\iota}\left[\left\|\int_{T} \iota(v) d \mu(v)\right\|^{2} / \int_{T}\|\iota(v)\|^{2} d \mu(v)\right] \in[0,1] .
$$

We then define

$$
\delta(T)=\sup _{\mu} \delta(\mu) \in[0,1] .
$$

Here, if we restrict the choices of $\mu$ to those with barycenter at a given $v \in T$, we denote the corresponding number by $\delta(T, v)$.

Theorem 3.7. Let $0 \leq \delta_{0}<1$, and let $y_{\leq \delta_{0}}$ denote the class of CAT( 0$)$ spaces $Y$ satisfying $\delta\left(T C_{p} Y\right) \leq \delta_{0}$ for all $p \in Y$. Let $\left\{G_{l}=\left(V_{l}, E_{l}\right)\right\}_{l \in L}$ be a sequence of finite connected graphs with $L$ an unbounded set of positive integers, satisfying the following conditions for some positive integer $d_{0}$, positive real number $\mu_{0}$ and $a$ choice of $\beta>1$ sufficiently close to 1 :

(i) $3 \leq \operatorname{deg}(u) \leq d_{0}$ for all $l \in L$ and all $u \in V_{l}$,

(ii) $\operatorname{girth}\left(G_{l}\right) \geq l$ and $\operatorname{diam}\left(G_{l}\right) \leq \mathrm{const} \cdot l$ for all $l \in L$,

(iii) $\lambda_{1}\left(G_{l}, \mathbb{R}\right) \geq \mu_{0}$ for all $l \in L$,

(iv) the number of embedded paths in $G_{l}$ of length less than $\frac{l}{2}$ is less than const $\cdot \beta^{l / 2}$.

Then a random group of the graph model associated with $\left\{G_{l}\right\}_{l \in L}$ is infinite hyperbolic and has property $F \mathcal{Y}_{\leq \delta_{0}}$.

Proof. If $Y \in \mathcal{y}_{\leq \delta_{0}}$, then by [11], Proposition 5.3,

$$
\lambda_{1}\left(G_{l}, T C_{p} Y\right) \geq\left(1-\delta\left(T C_{p} Y\right)\right) \lambda_{1}\left(G_{l}, \mathbb{R}\right) \geq\left(1-\delta_{0}\right) \lambda_{1}\left(G_{l}, \mathbb{R}\right)
$$

for all $p \in Y$. 
We now consider the sequence of graphs $\left\{G_{l^{\prime} / j}^{(j)}\right\}_{l^{\prime} \in j L}$ as in the previous subsection, where $j$ is chosen large enough so that the condition $\left(\mathrm{iv}^{\prime}\right)$ is satisfied for a choice of $\beta>1$ sufficiently close to 1 . The graph $G_{l^{\prime} / j}^{(j)}$ satisfies the condition (iii') : $\lambda_{1}\left(G_{l^{\prime} / j}^{(j)}, \mathbb{R}\right) \geq c\left(\mu_{0}, j\right)>0$.

Corollary 3.8. Let $0 \leq \delta_{0}<1$, and let $\mathcal{Y}_{\leq \delta_{0}}$ denote the class of CAT( 0$)$ spaces $Y$ satisfying $\delta\left(T C_{p} Y\right) \leq \delta_{0}$ for all $p \in Y$. Let $\left\{G_{l}=\left(V_{l}, E_{l}\right)\right\}_{l \in L}$ be a sequence of finite connected graphs with $L$ an unbounded set of positive integers, satisfying the following conditions for some positive integer $d_{0}$ and positive real number $\mu_{0}$ :

(i) $3 \leq \operatorname{deg}(u) \leq d_{0}$ for all $l \in L$ and all $u \in V_{l}$,

(ii) $\operatorname{girth}\left(G_{l}\right) \geq l$ and $\operatorname{diam}\left(G_{l}\right) \leq \mathrm{const} \cdot l$ for all $l \in L$,

(iii) $\lambda_{1}\left(G_{l}, \mathbb{R}\right) \geq \mu_{0}$ for all $l \in L$.

For each $l \in L$, let $G_{l}^{(j)}$ be the $j$-subdivision of $G_{l}$, and set $l^{\prime}=j l$. Here, $j$ is chosen large enough so that $\left\{G_{l^{\prime} / j}^{(j)}\right\}_{l^{\prime} \in j L}$ satisfies

(iv') the number of embedded paths in $G_{l^{\prime} / j}^{(j)}$ of length less than $\frac{l^{\prime}}{2}$ is less than const $\cdot \beta^{l^{\prime} / 2}$

for a choice of $\beta>1$ sufficiently close to 1 . Then a random group of the graph model associated with $\left\{G_{l^{\prime} / j}^{(j)}\right\}_{l^{\prime} \in j L}$ is infinite hyperbolic and has property $F y_{\leq \delta_{0}}$.

Proof. One has only to verify that $\lambda_{1}\left(G_{l^{\prime} / j}^{(j)}, T C_{p} Y\right)$ is bounded from below by a positive constant, independent of $l^{\prime}, Y$ and $p$. As was already noted, we have $\lambda_{1}\left(G_{l^{\prime} / j}^{(j)}, \mathbb{R}\right) \geq c\left(\mu_{0}, j\right)$ for all $l^{\prime} \in j L$. Therefore, as in the preceding proof,

$$
\lambda_{1}\left(G_{l^{\prime} / j}^{(j)}, T C_{p} Y\right) \geq\left(1-\delta_{0}\right) c\left(\mu_{0}, j\right),
$$

providing the desired estimate.

Remark 5. With the notations and assumptions as in Theorem 3.5, it is plausible that $\lambda_{1}\left(G_{l}^{(j)}, T C_{p} Y\right) \geq c\left(\lambda_{0}, j\right)>0$ holds for all $l \in L$, all $y \in \mathcal{Y}$ and all $p \in Y$. If this was the case, we would obtain a version of Theorem 3.5 for the sequence of graphs $\left\{G_{l^{\prime} / j}^{(j)}\right\} l^{\prime} \in j L$.

\section{Distortion and the invariant $\delta$}

In view of the assumption for CAT(0) spaces in Corollary 3.8, it is important to estimate the invariant $\delta$ of the tangent cones of a CAT(0) space. In this section, we first give an upper bound of $\delta\left(T, 0_{T}\right)$, where $T$ is a CAT(0) metric cone with cone point $0_{T}$, in terms of the radial distortion (defined below) of $T$. We then estimate the radial distortion of the tangent cones of some Euclidean buildings. 
We begin with some definitions.

Definition 4.1. (1) For a metric space $S$, let $T=C(S)=\left(S \times \mathbb{R}_{\geq 0}\right) /(S \times\{0\})$. Define a distance $d_{T}$ on $T$ by $d_{T}\left(v, v^{\prime}\right)=t^{2}+t^{\prime 2}-2 t t^{\prime} \cos \min \left\{d_{S}\left(u, u^{\prime}\right), \pi\right\}$, where $v=(u, t), v^{\prime}=\left(u^{\prime}, t^{\prime}\right) \in T$. The metric space $\left(T, d_{T}\right)$ is called the metric cone over $S$.

(2) Let $D_{\text {rad }}(T)$ denote the infimum number $D$ satisfying the following condition: there exists a map $\iota: T \rightarrow \mathscr{H}$, where $\mathscr{H}$ is a Hilbert space, such that

$$
\iota(v)=t \cdot \iota(u) \quad \text { with } \quad\|\iota(u)\|=1
$$

and

$$
\frac{1}{D} \cdot d_{T}\left(v, v^{\prime}\right) \leq\left\|\iota(v)-\iota\left(v^{\prime}\right)\right\| \leq d_{T}\left(v, v^{\prime}\right)
$$

for all $v=(u, t), v^{\prime}=\left(u^{\prime}, t^{\prime}\right) \in T$. If no such map exists, then we define $D_{\operatorname{rad}}(T)=$ $\infty$. The number $D_{\mathrm{rad}}(T)$ is called the radial distortion of $T$. Note that $D_{\mathrm{rad}}(T)$ is not less than the usual distortion (cf. [17]) of $T$.

Lemma 4.2. Let $T$ be a CAT(0) space, and $\mu$ a finite-support probability measure on $T$. Let $\iota: T \rightarrow \mathscr{H}$ be a 1-Lipschitz map, where $\mathscr{H}$ is a Hilbert space. Then we have

$$
\int_{T}\left\|\iota(v)-\overline{\iota_{*} \mu}\right\|^{2} d \mu(v) \geq \frac{1}{D(\iota)^{2}} \int_{T} d_{T}(v, \bar{\mu})^{2} d \mu(v),
$$

where $D(\iota)$ is the distortion of $\iota$, that is, the minimum number D such that (4.2) holds for all $v, v^{\prime} \in T$.

Proof. By using (2.3) and the fact that the inequality becomes an equality for a Hilbert space, we obtain

$$
\begin{aligned}
\int_{T}\left\|\iota(v)-\overline{\iota_{*} \mu}\right\|^{2} d \mu(v) & =\frac{1}{2} \int_{T}\|\iota(v)-\iota(w)\|^{2} d \mu(v) d \mu(w) \\
& \geq \frac{1}{D(\iota)^{2}} \cdot \frac{1}{2} \int_{T} d_{T}(v, w)^{2} d \mu(v) d \mu(w) \\
& \geq \frac{1}{D(\iota)^{2}} \int_{T} d_{T}(v, \bar{\mu})^{2} d \mu(v) .
\end{aligned}
$$

Proposition 4.3. Let $T$ be a $\mathrm{CAT}(0)$ metric cone with cone point $0_{T}$. Then we have

$$
\delta\left(T, 0_{T}\right) \leq 1-\frac{1}{D_{\operatorname{rad}}(T)^{2}} .
$$

Proof. Let $\iota: T \rightarrow \mathscr{H}$ be a map with the properties (4.1) and (4.2). Let $\mu$ be a finite-support probability measure on $T$ such that $\bar{\mu}=0_{T}$. Then

$$
\delta(\mu) \leq \frac{\left\|\int_{T} \iota(v) d \mu(v)\right\|^{2}}{\int_{T}\|\iota(v)\|^{2} d \mu(v)}=\frac{\left\|\overline{\iota_{*} \mu}\right\|^{2}}{\int_{T}\|\iota(v)\|^{2} d \mu(v)} .
$$


On the other hand,

$$
\int_{T}\left\|\iota(v)-\overline{\iota_{*} \mu}\right\|^{2} d \mu(v)=\int_{T}\|\iota(v)\|^{2} d \mu(v)-\left\|\overline{\iota_{*} \mu}\right\|^{2} .
$$

Therefore,

$$
\delta(\mu) \leq 1-\frac{\int_{T}\left\|\iota(v)-\overline{\iota_{*} \mu}\right\|^{2} d \mu(v)}{\int_{T}\|\iota(v)\|^{2} d \mu(v)} \leq 1-\frac{1}{D(\iota)^{2}}
$$

by Lemma 4.2, and the proposition follows.

Remark 6. It should be useful in future study to have an estimate of the Wang invariant $\lambda_{1}(G, T)$ from below. Indeed, we can show that

$$
\lambda_{1}(G, T) \geq \frac{1}{D(T)^{2}} \lambda_{1}(G, \mathbb{R}),
$$

where $D(T)$ denotes the (usual) distortion of $T$, holds for a finite connected graph $G$ and any CAT $(0)$ space $T$ which is not necessarily a cone. By the variance inequality (2.3), the denominator of (3.1) is estimated as

$$
\sum_{u \in V} v_{G}(u) d_{T}\left(\varphi(u), \operatorname{bar}\left(\varphi_{*} v_{G}\right)\right)^{2} \leq \frac{1}{2} \sum_{u, v \in V} v_{G}(u) v_{G}(v) d_{T}(\varphi(u), \varphi(v))^{2},
$$

and therefore,

$$
\operatorname{RQ}(\varphi) \geq \frac{\frac{1}{2} \sum_{u \in V} v_{G}(u) \sum_{v \in V} \mu_{G}(u, v) d_{T}(\varphi(u), \varphi(v))^{2}}{\frac{1}{2} \sum_{u, v \in V} v_{G}(u) v_{G}(v) d_{T}(\varphi(u), \varphi(v))^{2}} .
$$

Now suppose that $\iota: T \rightarrow \mathscr{H}$ is a map satisfying (4.2), where $\mathscr{H}$ is a Hilbert space. Then clearly,

the right-hand side of (4.4)

$$
\begin{aligned}
& \geq \frac{1}{D^{2}} \frac{\frac{1}{2} \sum_{u \in V} v_{G}(u) \sum_{v \in V} \mu_{G}(u, v)\|(\iota \circ \varphi)(u)-(\iota \circ \varphi)(v)\|^{2}}{\frac{1}{2} \sum_{u, v \in V} v_{G}(u) v_{G}(v)\|(\iota \circ \varphi)(u)-(\iota \circ \varphi)(v)\|^{2}} \\
& =\frac{1}{D^{2}} \frac{\frac{1}{2} \sum_{u \in V} v_{G}(u) \sum_{v \in V} \mu_{G}(u, v)\|(\iota \circ \varphi)(u)-(\iota \circ \varphi)(v)\|^{2}}{\sum_{u \in V} v_{G}(u)\left\|(\iota \circ \varphi)(u)-\operatorname{bar}\left((\iota \circ)_{*} v_{G}\right)\right\|^{2}} \\
& \geq \frac{1}{D^{2}} \lambda_{1}(G, \mathscr{H})=\frac{1}{D^{2}} \lambda_{1}(G, \mathbb{R}),
\end{aligned}
$$

and we conclude (4.3). Note that we have used the fact that the variance inequality (2.3) becomes an equality for a Hilbert space.

As mentioned in the Introduction, the fixed-point property for Euclidean buildings (of certain types) is of particular interest. In the remainder of this section, we will estimate the radial distortion of the tangent cones of some Euclidean buildings. 
A building is a simplicial complex which is the union of a family of subcomplexes, called apartments, satisfying a certain set of axioms (see [3]). One of the axioms requires that the apartments are isomorphic to a Coxeter complex of the same type. Here a Coxeter complex is a certain simplicial complex canonically associated with a Coxeter group; e.g., the Coxeter complex of the symmetric group $S_{n+1}$ is isomorphic to a triangulated $(n-1)$-sphere. A building is called Euclidean if its apartments are isomorphic to a Euclidean Coxeter complex; e.g., the Coxeter complex of type $\widetilde{A_{n}}$, which is associated with the group $S_{n+1} \ltimes\left(\mathbb{Z}^{n+1} / \mathbb{Z}(1, \ldots, 1)\right)$ and is isomorphic to a triangulated Euclidean $n$-space. A Euclidean building can be equipped with a distance by transplanting the Euclidean distance onto each apartment, and the building becomes a CAT(0) space with this distance (see [3], Chapter 6).

Henceforth, we restrict our attention to the Euclidean building associated with the simple algebraic group PGL $(n+1, \mathbb{Q} r)$, where $r$ is a prime and $\mathbb{Q} r$ is the $r$-adic number field. Let $Y_{n, r}$ denote this building; it is $n$-dimensional, and its apartments are simplicially isometric to the Euclidean Coxeter complex of type $\widetilde{A}_{n}$.

If $n=1, Y_{1, r}$ is a regular tree of degree $r+1$ with all edges having equal length. If $p$ is an interior point of an edge, then the tangent cone at $p$ is isometric to a line, whose radial distortion and $\delta$ take trivial values. Suppose that $p$ is a vertex. Then the tangent cone at $p$ is isometric to the $(r+1)$-pod $P_{r+1}$, which is the union of $r+1$ half-lines with all endpoints identified. The radial distortion of $P_{r+1}$ is realized by arranging it in $\mathbb{R}^{r}$ so that the half-lines pass through the vertices of a regular $r$ simplex, and thus $D_{\text {rad }}\left(P_{r+1}\right)=\sqrt{2 r /(r+1)}$. On the other hand, $\delta\left(P_{r+1}\right)=0$ as verified in [11], p. 172, Example 3.

If $n=2, Y_{2, r}$ is two-dimensional, and its apartments are simplicially isometric to the Euclidean plane with equilateral triangulation. Simplicially, the links of its vertices are all isomorphic to the same generalized triangle of degree $r+1$, which is a regular bipartite graph of degree $r+1$ with $2\left(r^{2}+r+1\right)$ vertices and will be denoted by $\mathscr{g}_{r}$. Metrically, this means that the tangent cone at $p \in Y_{2, r}$ is isometric to a Euclidean plane if $p$ is an interior point of a maximal simplex, to the product of $(r+1)$-pod $P_{r+1}$ with a line if $p$ is an interior point of an edge, and to the metric cone $C\left(\mathscr{G}_{r}\right)$ over the graph $\mathscr{G}_{r}$ equipped with a distance by assigning length $\pi / 3$ to each edge if $p$ is a vertex. In the first case the values of the invariants in question are trivial, while in the second case they are identical to those of $P_{r+1}$. Therefore, it remains to examine the third case that $p$ is a vertex of $Y_{2, r}$, in which case it is known [11] that

$$
\delta\left(C\left(\mathscr{G}_{r}\right)\right) \geq \frac{(\sqrt{r}-1)^{2}}{2(r-\sqrt{r}+1)} .
$$

In fact, let $\mu_{0}$ be the probability measure on $C\left(\mathscr{E}_{r}\right)$ given by $\mu_{0}=\sum_{i=1}^{N} \frac{1}{N} \operatorname{Dirac}_{e_{i}}$, where $N=2\left(r^{2}+r+1\right)$ and $e_{i}, i=1, \ldots, N$, are the vertices of $\varepsilon_{r}$. Then the barycenter of $\mu_{0}$ coincides with the cone point of $C\left(\mathscr{E}_{r}\right)$, and we showed that

$$
\delta\left(\mu_{0}\right)=\frac{(\sqrt{r}-1)^{2}}{2(r-\sqrt{r}+1)} .
$$


In order to verify the ' $\leq$ '-part of this equality, we [11], §7, introduced a certain family of 1-Lipschitz embeddings of the cone $C\left(\mathscr{G}_{r}\right)$ into Euclidean spaces. We now recall these embeddings and then use them to estimate the radial distortion of $C\left(\mathscr{E}_{r}\right)$.

Let $V=\bigoplus_{i=1}^{N} \mathbb{R} e_{i}$ be the real vector space having the vertices $e_{i}$ as formal basis vectors. Note that there is a natural inclusion $C\left(\mathscr{G}_{r}\right) \hookrightarrow V$. We consider all positive semidefinite inner products $\langle\cdot, \cdot\rangle$ on $V$ whose value $\left\langle e_{i}, e_{j}\right\rangle$ on each pair of vertices $e_{i}, e_{j}$ depends only on the combinatorial distance $d \boldsymbol{g}_{r}\left(e_{i}, e_{j}\right)$ between these vertices. We also require that the inner products of adjacent vertices are the same as those in $C\left(\mathscr{G}_{r}\right)$. Thus we consider symmetric bilinear forms $\langle\cdot, \cdot\rangle_{a, b}$ on $V$ defined by

$$
\left\langle e_{i}, e_{j}\right\rangle_{a, b}= \begin{cases}1 & \text { if } d \boldsymbol{g}_{r}\left(e_{i}, e_{j}\right)=0, \\ 1 / 2 & \text { if } d \boldsymbol{g}_{r}\left(e_{i}, e_{j}\right)=1, \\ a & \text { if } d \boldsymbol{g}_{r}\left(e_{i}, e_{j}\right)=2, \\ b & \text { if } d \boldsymbol{g}_{r}\left(e_{i}, e_{j}\right)=3,\end{cases}
$$

and restrict the parameters $a, b$ to the range where $\langle\cdot, \cdot\rangle_{a, b}$ is positive semidefinite. For $a, b$ in this range, consider the sum of the eigenspaces belonging to the positive eigenvalues of the Gram matrix $G_{a, b}=\left(\left\langle e_{i}, e_{j}\right\rangle_{a, b}\right)$, and let $W_{a, b}$ be the corresponding subspace of $V$. Restricted on $W_{a, b}$ the inner product $\langle\cdot, \cdot\rangle_{a, b}$ is positive definite, and the natural projection $V \rightarrow W_{a, b}$ preserves the inner products. The composition of the maps $C\left(\mathscr{G}_{r}\right) \hookrightarrow V \rightarrow W_{a, b}$ gives a map from $C\left(\mathscr{E}_{r}\right)$ into the Euclidean space $W_{a, b}$. We denote this map by $\iota_{a, b}$; it is radial and 1-Lipschitz; it is also isometric when restricted to the cone over each edge of $\mathscr{G}_{r}$.

It is easy to see that the distortion of $\iota_{a, b}$ is computed as

$$
\begin{aligned}
D\left(\iota_{a, b}\right) & =\max \left\{\sqrt{\frac{2-2 \cos (2 \pi / 3)}{2-2 a}}, \sqrt{\frac{2-2 \cos \pi}{2-2 b}}\right\} \\
& =\max \left\{\sqrt{\frac{3}{2-2 a}}, \sqrt{\frac{2}{1-b}}\right\} .
\end{aligned}
$$

On the other hand, $G_{a, b}$ can be readily related to the adjacency matrix of $\mathscr{E}_{r}$, whose eigenvalues were computed by Feit and Higman [4]. It follows that the eigenvalues of $G_{a, b}$ are given by

$$
\left(r^{2}+r+1\right)(a \pm b)+(1-a) \pm(1 / 2-b)(r+1)
$$

with multiplicities 1 and

$$
(1-a) \pm(1 / 2-b) \sqrt{r}
$$

with multiplicities $r^{2}+r$. Under the constraint that these are nonnegative, the quantity (4.5) takes its minimum with

$$
a=\frac{r-1-\sqrt{r}}{2 r}, \quad b=\frac{r^{2}-r-(r+1) \sqrt{r}}{2 r^{2}} .
$$


(Incidentally, these values coincide with those giving the optimal upper bound of $\delta\left(\mu_{0}\right)$.) The minimum value $2 r / \sqrt{(r+1)(r+\sqrt{r})}$ gives an upper bound of $D_{\text {rad }}\left(C\left(\mathscr{E}_{r}\right)\right)$. Note, in particular, that $D_{\text {rad }}\left(C\left(\mathscr{E}_{r}\right)\right)<2$ for all primes $r$. With the above values of $a, b, G_{a, b}$ has positive eigenvalues $r^{2}+1-(r+1) \sqrt{r}$, $(r+1+\sqrt{r}) / r$ with multiplicities $1, r^{2}+r$ respectively and zero eigenvalue with multiplicity $r^{2}+r+1$. Therefore, $W_{a, b}$ has dimension $r^{2}+r+1$. Observe that as $r$ tends to infinity, the above values of $a, b$ both approach $1 / 2$. This means that when $r$ is large, the images of the vertices of $\mathscr{E}_{r}$ in $W_{a, b}$ are nearly at equidistance to one another.

We now treat the case of general $n$. Analogously to the $n=2$ case, if $p \in Y_{n, r}$ is not a vertex, then the tangent cone of $Y_{n, r}$ at $p$ is isometric to a metric cone of the form $\prod_{i=1}^{m} T_{k_{i}, r} \times \mathbb{R}^{l}$, where $T_{k_{i}, r}$ is the tangent cone of $Y_{k_{i}, r}$ at a vertex, $l>0$ and $\sum_{i=1}^{m} k_{i}+l=n$. Since $D_{\mathrm{rad}}\left(\prod_{i=1}^{m} T_{k_{i}, r} \times \mathbb{R}^{l}\right)=\max _{1 \leq i \leq m} D_{\mathrm{rad}}\left(T_{k_{i}, r}\right)$, we assume henceforth that $p$ is a vertex of $Y_{n, r}$. Then the tangent cone at $p$ is isometric to the metric cone $C\left(S_{n, r}\right)$ over the spherical building $S_{n, r}$ associated with the finite group PGL $\left(n+1, \mathbb{F}_{r}\right)$, and the apartments of $S_{n, r}$ are simplicially isometric to the tessellated unit $(n-1)$-sphere associated with the symmetric group $S_{n+1}$. A chamber of $S_{n, r}$ has $n$ vertices $e_{1}, \ldots, e_{n}$, and the distances between them measured by the metric of $C\left(S_{n, r}\right)$ are given by

$$
d_{C\left(s_{n, r}\right)}\left(e_{i}, e_{j}\right)=\sqrt{2-2 \sqrt{[i(n+1-j)] /[j(n+1-i)]}}
$$

when the vertices are appropriately ordered. The minimum of these distances is

$$
d_{\min }= \begin{cases}\sqrt{2-2 \sqrt{(n-1) /(n+3)}} & \text { if } n \text { is odd } \\ \sqrt{2-2 n /(n+2)}=2 / \sqrt{n+2} & \text { if } n \text { is even }\end{cases}
$$

Motivated by the observation we made at the end of the preceding paragraph, we construct an embedding of $C\left(S_{n, r}\right)$ into a Euclidean space as follows. Denote the number of vertices of $S_{n, r}$ by $N$. First take a regular simplex $\sigma$ with $N$ vertices in $\mathbb{R}^{N}$ whose vertices are located in the unit sphere with center at the origin and at the distance $d_{\min }$ to one another. Next map one by one the vertices of $\delta_{n, r}$ to those of $\sigma$, and then extend it naturally to a radial embedding of $C\left(S_{n, r}\right)$. Clearly, this embedding, which we call $\iota$, is 1-Lipschitz and has the same distortion as that of its restriction to $S_{n, r}$. To estimate the distortion, we have to bound the ratio $d_{C\left(S_{n, r}\right)}\left(v, v^{\prime}\right) /\left\|\iota(v)-\iota\left(v^{\prime}\right)\right\|$ from above over all pairs of distinct points $v, v^{\prime}$ in $\delta_{n, r}$. It is easy to see that if we vary $v, v^{\prime}$, the above ratio is maximized when they are at vertices of $\delta_{n, r}$. Since $\delta_{n, r}$ is a building, we may also assume that $v, v^{\prime}$ are in the same apartment of $\delta_{n, r}$. Therefore, the problem is reduced to bounding $d_{C\left(S_{n, r}\right)}\left(v, v^{\prime}\right) /\left\|\iota(v)-\iota\left(v^{\prime}\right)\right\|$ from above over all pairs of distinct vertices $v, v^{\prime}$ in a fixed apartment of $S_{n, r}$. Now this ratio is clearly bounded from above by $2 / d_{\min }$, which therefore gives an upper bound of the distortion of $\iota$. Note that the constant $2 / d_{\min }$ is monotone increasing with $n$, and diverges to infinity as $n \rightarrow \infty$. 
We record the consequence of the preceding discussion as

Proposition 4.4. The radial distortion and the invariant $\delta$ of all tangent cones of the Euclidean building $Y_{n, r}$ are bounded from above by

$$
\begin{cases}2 / \sqrt{2-2 \sqrt{(n-1) /(n+3)}} & \text { if } n \text { is odd, } \\ \sqrt{n+2} & \text { if } n \text { is even, }\end{cases}
$$

and

$$
\begin{cases}(2+2 \sqrt{(n-1) /(n+3)}) / 4 & \text { if } n \text { is odd } \\ (n+1) /(n+2) & \text { if } n \text { is even }\end{cases}
$$

respectively.

Proof. Let $T$ be a tangent cone of $Y_{n, r}$. Then $D_{\text {rad }}(T)$ is bounded as stated, and so is $\delta\left(T, 0_{T}\right)$. For $v \neq 0_{T}$, the tangent cone $T C_{v} T$ is isometric to the product of lower dimensional cones and possibly a Euclidean space. Since $\delta(T, v) \leq \delta\left(T C_{v} T, 0_{v}\right)$ (see [11], Lemma 6.2) and the invariant $\delta$ behaves in the same way as the radial distortion for the product, we conclude that $\delta(T, v)$ and hence $\delta(T)$ is also bounded as stated.

Remark 7. As noted above, the upper bound in the proposition diverges to infinity as $n \rightarrow \infty$. The embedding $\iota$ does not preserve the shape of chambers of $\delta_{n, r}$, and one might expect that by constructing an embedding so that it preserves the shape of chambers of $S_{n, r}$, one would get a better upper bound. However, numerical test done for $n=3$ indicates that the upper bound so obtained should diverge to infinity as $r \rightarrow \infty$ even though $n$ is kept bounded.

Combining the proposition above with Corollary 3.8, we obtain

Theorem 4.5. For a fixed positive integer $N$, let $\mathscr{B}_{\leq N}$ denote the family of all the Euclidean buildings $Y_{n, r}$ with $n \leq N$ and $r$ arbitrary prime. Let $\left\{G_{l}=\left(V_{l}, E_{l}\right)\right\}_{l \in L}$ be a sequence of finite connected graphs, with $L$ an unbounded set of positive integers, satisfying the following conditions for some positive integer $d_{0}$ and positive real number $\mu_{0}$ :

(i) $3 \leq \operatorname{deg}(u) \leq d_{0}$ for all $l \in L$ and all $u \in V_{l}$,

(ii) $\operatorname{girth}\left(G_{l}\right) \geq l$ and $\operatorname{diam}\left(G_{l}\right) \leq \mathrm{const} \cdot l$ for all $l \in L$,

(iii) $\lambda_{1}\left(G_{l}, \mathbb{R}\right) \geq \mu_{0}$ for all $l \in L$.

For each $l \in L$, let $G_{l}^{(j)}$ be the $j$-subdivision of $G_{l}$, and set $l^{\prime}=j l$. Here, $j$ is chosen large enough so that $\left\{G_{l^{\prime} / j}^{(j)}\right\}_{l^{\prime} \in j L}$ satisfies

$\left(\mathrm{iv}^{\prime}\right)$ the number of embedded paths in $G_{l^{\prime} / j}^{(j)}$ of length less than $\frac{l^{\prime}}{2}$ is less than const $\cdot \beta^{l^{\prime} / 2}$ 
for a choice of $\beta>1$ sufficiently close to 1 . Then a random group of the graph model associated with $\left\{G_{l^{\prime} / j}^{(j)}\right\}_{l^{\prime} \in j L}$ is infinite hyperbolic and has property $F \mathscr{B}_{\leq N}$.

\section{Appendix}

In this Appendix, we will prove Proposition 3.4. Let $G=(V, E)$ be a finite connected graph, and $Y$ a CAT(0) space. For a map $\varphi: V \rightarrow Y$ and a positive integer $n$, the $n$-step energy of $\varphi$ is defined by

$$
E_{\mu_{G}^{n}}(\varphi)=\frac{1}{2} \sum_{u \in V} v_{G}(u) \sum_{v \in V} \mu_{G}^{n}(u, v) d_{Y}(\varphi(u), \varphi(v))^{2},
$$

where $\mu_{G}$ is the standard random walk on $G$ and $\nu_{G}$ is the standard probability measure on $V$ (cf. §3.1). We have the following

Lemma 5.1. For any map $\varphi: V \rightarrow Y$ and any positive integer $n$, we have

$$
E_{\mu_{G}^{n}}(\varphi) \leq \frac{2}{\lambda_{1}(G, Y)} E_{\mu_{G}}(\varphi)
$$

Proof. Let $\bar{\varphi}=\operatorname{bar}\left(\varphi_{*} \nu_{G}\right)$. Using the triangle inequality and the symmetry of $\mu_{G}^{n}$ with respect to $v_{G}$, we obtain

$$
\begin{aligned}
E_{\mu_{G}^{n}}(\varphi) & \leq \frac{1}{2} \sum_{u \in V} v_{G}(u) \sum_{v \in V} \mu_{G}^{n}(u, v)\left(d_{Y}(\varphi(u), \bar{\varphi})+d_{Y}(\varphi(v), \bar{\varphi})\right)^{2} \\
& \leq \frac{1}{2} \sum_{u \in V} v_{G}(u) \sum_{v \in V} \mu_{G}^{n}(u, v)\left(2 d_{Y}(\varphi(u), \bar{\varphi})^{2}+2 d_{Y}(\varphi(v), \bar{\varphi})^{2}\right) \\
& =2 \sum_{u \in V} v_{G}(u) \sum_{v \in V} \mu_{G}^{n}(u, v) d_{Y}(\varphi(u), \bar{\varphi})^{2} \\
& =2 \sum_{u \in V} v_{G}(u) d_{Y}(\varphi(u), \bar{\varphi})^{2} .
\end{aligned}
$$

On the other hand, by the definition of $\lambda_{1}(G, Y)$, we have

$$
\sum_{u \in V} v_{G}(u) d_{Y}(\varphi(u), \bar{\varphi})^{2} \leq \frac{1}{\lambda_{1}(G, Y)} E_{\mu_{G}}(\varphi) .
$$

Combining (5.1) and (5.2), we obtain the desired inequality.

Let $\Gamma=F_{k}$ be the free group generated by $S=\left\{s_{1}^{ \pm}, \ldots, s_{k}^{ \pm}\right\}$, and let $\Gamma$ act on itself from the left. Let $\alpha: \vec{E} \rightarrow S$ be an $S$-labelling of $G$. Recall that associated with $\alpha$ is the group $\Gamma_{\alpha}=\Gamma / \overline{R_{\alpha}}$, where $R_{\alpha}=\{\alpha(\vec{c}) \mid \vec{c}$ is a cycle in $G\}$ and $\overline{R_{\alpha}}$ is its normal closure. As in [21], we will exclusively work on $\Gamma$ rather than on $\Gamma_{\alpha}$. 
For each positive integer $n$, define the 'push-forward' of $\mu_{G}^{n}$ with respect to $\alpha$ by

$$
\bar{\mu}_{\Gamma, \alpha}^{n}\left(\gamma, \gamma^{\prime}\right)=\sum_{u \in V} v_{G}(u) \sum_{\substack{|\vec{p}|=n, p_{0}=u \\ \gamma \alpha(\vec{p})=\gamma^{\prime}}} \mu_{G}^{n}(\vec{p})=\sum_{\substack{|\vec{p}|=n \\ \gamma \alpha(\vec{p})=\gamma^{\prime}}} v_{G}\left(p_{0}\right) \mu_{G}^{n}(\vec{p}),
$$

where $p_{0}$ is the initial vertex of $\vec{p}$. Note that $\bar{\mu}_{\Gamma, \alpha}^{n}$ is a $\Gamma$-invariant random walk on $\Gamma$. For a homomorphism $\rho^{(\alpha)}: \Gamma_{\alpha} \rightarrow \operatorname{Isom}(Y)$ and a $\rho^{(\alpha)}$-equivariant map $f^{(\alpha)}: \Gamma_{\alpha} \rightarrow$ $Y$, set $\rho=\rho^{(\alpha)} \circ$ pr and $f=f^{(\alpha)} \circ \mathrm{pr}$, where pr is the natural projection from $\Gamma$ onto $\Gamma_{\alpha}$. Then $f$ is a $\rho$-equivariant map, for which we can transplant the estimate of Lemma 5.1 to obtain

$$
E_{\bar{\mu}_{\Gamma, \alpha}^{n}}(f) \leq \frac{2}{\lambda_{1}(G, Y)} E_{\bar{\mu}_{\Gamma, \alpha}}(f)
$$

for all positive integers $n$ (cf. [21], p. 155-156).

For $n, \gamma, \gamma^{\prime}$ fixed, regard $\bar{\mu}_{\Gamma, \alpha}^{n}\left(\gamma, \gamma^{\prime}\right)$ as a random variable of $\alpha$, and denote its expectation by $\bar{\mu}_{\Gamma, G}^{n}\left(\gamma, \gamma^{\prime}\right)$. We have the following lemma, which compares $\bar{\mu}_{\Gamma, G}^{n}$ with the standard random walk $\mu_{\Gamma}$ on $\Gamma$, given by

$$
\mu_{\Gamma}\left(\gamma, \gamma^{\prime}\right)= \begin{cases}\frac{1}{2 k} & \text { if } \gamma^{\prime}=\gamma s \text { for some } s \in S \\ 0 & \text { otherwise. }\end{cases}
$$

Lemma 5.2 (cf. [21], Lemma 2.12). Suppose that $\operatorname{deg}(u) \geq 2$ for all $u \in V$, and choose a positive integer $n$ so that $n<\operatorname{girth}(G) / 2$. Then there exist weights $P_{G}^{n}(l) \geq$ 0 with $\sum_{l=0}^{n} P_{G}^{n}(l)=1$, independent of $\gamma, \gamma^{\prime}$, such that

$$
\bar{\mu}_{\Gamma, G}^{n}\left(\gamma, \gamma^{\prime}\right)=\sum_{l=0}^{n} P_{G}^{n}(l) \mu_{\Gamma}^{l}\left(\gamma, \gamma^{\prime}\right) .
$$

Moreover, there exists an absolute constant $C<1$ such that

$$
Q_{G}^{n}:=\sum_{l \leq \sqrt{n}} P_{G}^{n}(l) \leq C
$$

unless $n=1$.

Proof. Note that any ball of radius $n$ in $G$ is a tree; this is the most fundamental fact for the whole proof. The former part of the lemma can be proved by following Silberman's argument almost verbatim, and the weights $P_{G}^{n}(l)$ are given by

$$
P_{G}^{n}(l)=\sum_{u \in V} v_{G}(u) P_{G, u}^{n}(l),
$$

where $P_{G, u}^{n}(l)$ is the probability that an $n$-step random walk starting from $u$ reaches a vertex at distance $l$ from $u$. Here we prove the latter part of the lemma by an argument 
simpler than that proposed by Silberman. To do this, consider the standard Bernoulli walk on $\mathbb{Z}$, and let $b^{n}(r)$ denote the probability that an $n$-step walk starting from zero reaches an integer less than or equal to $r$ in absolute value. Since $\operatorname{deg}(u) \geq 2$ for all $u \in V$, the random walk on $G$ travels further than the Bernoulli walk on $\mathbb{Z}$. More precisely, we have

$$
\sum_{l \leq \sqrt{n}} P_{G, u}^{n}(l) \leq b^{n}(\sqrt{n}) .
$$

We now recall that the $n$-step Bernoulli walk has variance $n$. Then by the central limit theorem, we obtain

$$
b^{n}(\sqrt{n}) \underset{n \rightarrow \infty}{\rightarrow} \int_{-1}^{1} \frac{1}{\sqrt{2 \pi}} e^{-x^{2} / 2} d x<1 .
$$

Therefore, there exists $C<1$ such that

$$
\sum_{l \leq \sqrt{n}} P_{G, u}^{n}(l) \leq C
$$

for all $n$ (other than 1). Averaging over $u$, we conclude the latter assertion of the lemma.

The following lemma also can be proved by going on the same lines as Silberman's proof of [21], Lemma 2.13, which applies a general result on the concentration of measure to the random variable $\bar{\mu}_{\Gamma, \alpha}^{n}\left(\gamma, \gamma^{\prime}\right)$ defined on the set all $S$-labellings $\alpha$.

Lemma 5.3 (cf. [21], Lemma 2.13). In addition to the assumptions of Lemma 5.2, suppose that $\operatorname{deg}(u) \leq d$ for all $u \in V$. Then with probability at least $1-a_{1} e^{-a_{2}|V|}$, where $a_{1}=a_{1}(k, n), a_{2}=a_{2}(k, d, n)$, we have

$$
\bar{\mu}_{\Gamma, \alpha}^{n}\left(\gamma, \gamma^{\prime}\right) \geq \frac{1}{2} \bar{\mu}_{\Gamma, G}^{n}\left(\gamma, \gamma^{\prime}\right) \text { and } \bar{\mu}_{\Gamma, \alpha}\left(\gamma, \gamma^{\prime}\right) \leq \mu_{\Gamma}\left(\gamma, \gamma^{\prime}\right)
$$

for all $\gamma, \gamma^{\prime} \in \Gamma$.

With the ingredients above, the proof of Proposition 3.4 proceeds as in [21], proof of Proposition 2.14. We include it for the sake of completeness.

Proof of Proposition 3.4. If follows from Lemma 5.3 that

$$
E_{\bar{\mu}_{\Gamma, \alpha}^{n}}(f) \geq \frac{1}{2} E_{\bar{\mu}_{\Gamma, G}^{n}}(f), \quad E_{\bar{\mu}_{\Gamma, \alpha}}(f) \leq E_{\mu_{\Gamma}}(f)
$$

hold with the probability as in the statement of the proposition. By combining this with (5.3), we obtain

$$
E_{\bar{\mu}_{\Gamma, G}^{n}}(f) \leq \frac{4}{\lambda_{1}(G, Y)} E_{\mu_{\Gamma}}(f)
$$


with the same probability. By Lemma 5.2, we can estimate $E_{\bar{\mu}_{\Gamma, G}^{n}}(f)$ from below:

$$
\begin{aligned}
E_{\bar{\mu}_{\Gamma, G}^{n}}(f) & =\sum_{l=0}^{n} P_{G}^{n}(l) E_{\mu_{\Gamma}^{l}}(f) \\
& \geq \sum_{\sqrt{n}<l \leq n} P_{G}^{n}(l) E_{\mu_{\Gamma}^{l}}(f) \\
& \geq\left(\sum_{\sqrt{n}<l \leq n} P_{G}^{n}(l)\right) E_{\mu_{\Gamma}^{l_{0}}}(f) .
\end{aligned}
$$

Here $E_{\mu_{\Gamma}^{l_{0}}}(f)=\min \left\{E_{\mu_{\Gamma}^{l}}(f) \mid \sqrt{n}<l \leq n\right\}$. We also have $\sum_{\sqrt{n}<l \leq n} P_{G}^{n}(l)=$ $1-Q_{G}^{n} \geq 1-C$. Therefore, we conclude that

$$
E_{\mu_{\Gamma}^{l_{0}}}(f) \leq \frac{1}{1-C} \frac{4}{\lambda_{1}(G, Y)} E_{\mu_{\Gamma}}(f)
$$

holds with high probability.

Added in proof. During the submission of the present paper, we learned that Naor and Silberman [19] proved that the graph-model random group has the fixed-point property for a family of $p$-uniformly convex geodesic metric spaces with a certain Poincaré-type constant uniformly bounded. For a family of CAT(0) spaces (which are 2-uniformly convex), this condition is equivalent to the uniformly-boundedness of the Wang invariant (the condition (iii) in Theorem 3.5). However, our Theorem 4.5, the fixed-point theorem for a family of Euclidean buildings with dimensions bounded from above, does not follow from their result.

Let $y_{<1}$ denote the class of CAT(0) spaces $Y$ satisfying $\sup _{p \in Y} \delta\left(T C_{p} Y\right)<1$, which contains all of the Euclidean buildings $Y_{n, r}$ (cf. $\$ 4$ for the notation). By using our Corollary 3.8, it is shown that the group of Theorem 7.7 of [1], called the Gromov monster, has the fixed-point property for $y_{<1}$. It should be mentioned that the Gromov monster has the fixed-point property for a larger class of metric spaces, as shown by combining Theorem 1.2 of [19] with Theorem 7.7 of [1]. It is also worthwhile to mention that Kondo [14] has found examples of CAT(0) space $Y$ for which $\sup _{p \in Y} \delta\left(T C_{p} Y\right)=1$.

\section{References}

[1] G. Arzhantseva and T. Delzant, Examples of random groups. Preprint 2008, revised version (August 26, 2011). http://www.unige.ch/math/folks/arjantse/publicationsGA.html

[2] M. R. Bridson and A. Haefliger, Metric spaces of non-positive curvature. Grundlehren Math. Wiss. 319, Springer-Verlag, Berlin 1999. Zbl 0988.53001 MR 1744486

[3] K. S. Brown, Buildings. Springer-Verlag, New York 1989. Zbl 0715.20017 MR 969123 
[4] W. Feit and G. Higman, The nonexistence of certain generalized polygons. J. Algebra 1 (1964), 114-131. Zbl 0126.05303 MR 0170955

[5] E. Ghys, Groupes aléatoires [d'après Misha Gromov,...]. Astérisque 294 (2004), 173-204. Zbl 1134.20306 MR 2111644

[6] M. Gromov, Hyperbolic groups. In Essays in group theory, Math. Sci. Res. Inst. Publ. 8, Springer-Verlag, New York 1987, 75-263. Zbl 0634.20015 MR 0919829

[7] M. Gromov, Geometric group theory (Sussex, 1991), vol. 2: Asymptotic invariants of infinite groups. London Math. Soc. Lecture Note Ser. 182, Cambridge University Press, Cambridge 1993. Zbl 0841.20039 MR 1253544

[8] M. Gromov, Random walk in random groups. Geom. Funct. Anal. 13 (2003), 73-146. Zbl 1122.20021 MR 1978492

[9] H. Izeki, A fixed-point property of finitely generated groups and an energy of equivariant maps. In Probabilistic approach to geometry, Adv. Stud. Pure Math. 57, Math. Soc. Japan, Tokyo 2010, 171-188. Zbl 1201.58014 MR 2648259

[10] H. Izeki, T. Kondo, and S. Nayatani, Fixed-point property of random groups. Ann. Global Anal. Geom. 35 (2009), 363-379. Zbl 1236.20046 MR 2506240

[11] H. Izeki and S. Nayatani, Combinatorial harmonic maps and discrete-group actions on Hadamard spaces. Geom. Dedicata 114 (2005), 147-188. Zbl 1108.58014 MR 2174098

[12] J. Jost, Nonlinear Dirichlet forms. In New directions in Dirichlet forms, AMS/IP Stud. Adv. Math. 8, Amer. Math. Soc., Providence, RI, 1998, 1-47. Zbl 0914.31006 MR 1652278

[13] T. Kondo, Fixed-point theorems for random groups. In Probabilistic approach to geometry, Adv. Stud. Pure Math. 57, Math. Soc. Japan, Tokyo 2010, 263-272. Zbl 1239.20081 MR 2648264

[14] T. Kondo, CAT(0) spaces and expanders. Math. Z. 271 (2012), 343-355. Zbl 06050598 MR 2917147

[15] N. J. Korevaar and R. M. Schoen, Sobolev spaces and harmonic maps for metric space targets. Comm. Anal. Geom. 1 (1993), 561-659. Zbl 0862.58004 MR 1266480

[16] A. Lubotzky, R. Phillips, and P. Sarnak, Ramanujan graphs. Combinatorica 8 (1988), 261-277. Zbl 0661.05035 MR 963118

[17] J. Matoušek, Lectures on discrete geometry. Graduate Texts in Math. 212, SpringerVerlag, New York 2002. Zbl 0999.52006 MR 1899299

[18] U. F. Mayer, Gradient flows on nonpositively curved metric spaces and harmonic maps. Comm. Anal. Geom. 6 (1998), 199-253. Zbl 0914.58008 MR 1651416

[19] A. Naor and L. Silberman, Poincaré inequalities, embeddings, and wild groups. Compositio Math. 147 (2011), 1546-1572. Zbl 05961179 MR 2834732

[20] Y. Ollivier, A January 2005 invitation to random groups. Ensaios Matemáticos 10, Sociedade Brasileira de Matemática, Rio de Janeiro 2005. Zbl 1163.20311 MR 2205306

[21] L. Silberman, Addendum to: "Random walk in random groups" by M. Gromov. Geom. Funct. Anal. 13 (2003), 147-177. Zbl 1124.20027 MR 1978493

[22] M.-T. Wang, Generalized harmonic maps and representations of discrete groups. Comm. Anal. Geom. 8 (2000), 545-563. Zbl 0977.58018 MR 1775138 
Received October 16, 2009; revised October 11, 2011

H. Izeki, Department of Mathematics, Faculty of Science and Technology, Keio University, Kohoku-ku, Yokohama 223-8522, Japan

E-mail: izeki@math.keio.ac.jp

T. Kondo, Department of Mathematics, Graduate School of Science, Kobe University, Kobe 657-8501, Japan; current address: Mathematical Institute, Tohoku University, Sendai 9808578, Japan

E-mail: takefumi@math.tohoku.ac.jp

S. Nayatani, Graduate School of Mathematics, Nagoya University, Chikusa-ku, Nagoya 464-8602, Japan

E-mail: nayatani@math.nagoya-u.ac.jp 